(RESEARCH ARTICLE)

\title{
Risk factors and knowledge assessment of general public for chronic kidney disease in Taif Region, Saudi Arabia
}

\author{
Shaheen Sultana *, Rawan Alqurashi, Thekra Alqarni, Maram Alnefaie, Afnan kalakattawi, Rawan Alzahrani \\ Assistant Professor, Department of Pharmaceutics, College of Pharmacy, Taif University, Postal Code: 21974, P.O.Box: 888.
}

Publication history: Received on 07 October 2020; revised on 22 October 2020; accepted on 24 October 2020

Article DOI: https://doi.org/10.30574/wjarr.2020.8.1.0373

\begin{abstract}
Introduction: Chronic kidney disease (CKD) is a worldwide public health problem since the rapid increasing prevalence of relevant risk factors, like diabetes, hypertension, unhealthy diet, low to no physical activity and metabolic syndrome. The current study aimed at assessing the risk factors and the general public awareness of CKD in Taif region, Saudi Arabia.

Method: We conducted a cross-sectional study during the period of September 2019 to March 2020. For risk factors assessment data regarding CKD cases were collected from the department of nephrology at AL-Hada Military hospital in Taif. An online-based questionnaire was distributed to the general public for knowledge assessment of CKD. $6^{\text {th }}$ year pharmacy student at Taif University and nephrologists from the hospital were also invited to answer the questionnaire.

Results: The data of 456 CKD patients with 600 non-CKD individuals were compared for assessment of the most common risk factors. $99 \%$ of participants were CKD stage III-V patients. The majority, $n=371(81.57 \%)$, of the participants were $>50$ years old. $\mathrm{N}=328(71.93 \%$ ) had type 2 diabetes mellitus (Risk ratio= 2.32, CI= 2.03-2.65; Odd ratio=5.7, $\mathrm{CI}=4.36-7.45), \mathrm{n}=396(86.84 \%$ ) had hypertension (Risk ratio =9.65,CI-7.46-12.48; Odd ratio: $66.73, \mathrm{CI}=45.19$ 98.54) and $n=264(57.89 \%)$ had a history of heart diseases (Risk ratio=3.62, CI=2.96-4.42; Odd ratio= 7.22, CI= 5.429.62). In CKD groups, $28.07 \%$ of them had low physical activity. In contrast with the control group, only $4 \%$ had low physical activity level in their daily routine $(\mathrm{p}<0.00001)$. In the general public $(\mathrm{n}=1419), 919$ (64.7\%) had poor knowledge, 273 (19.22\%) had fair knowledge, and 227 respondents (16.01\%) had good knowledge of CKD.

Conclusion: The finding suggests that age $>50$ years, low educational attainment, family history of CKD, diabetes, hypertension, history of kidney stone, CVD and high BMI were significantly associated with CKD progression. We also found that the majority of the general public had poor knowledge of CKD compared to pharmacy graduates and nephrologists. These findings warrant the need for the development of awareness initiatives and programs designed to increase the level of knowledge of the general public of CKD.
\end{abstract}

Keywords: Chronic renal failure; Risk factor; Knowledge; Saudi Arabia; Diabetes

\section{Introduction}

Chronic kidney disease (CKD) has been a global public health issue in the past decades and affects more than $10 \%$ of the population worldwide. CKD is a progressive loss in renal function over a period of three months or years associated with several risk factors. Once kidneys are damaged, they cannot filter blood or perform other activities, which are usually associated with a reduction in glomerular filtration rate (GFR) and proteinuria; which later on progresses,

${ }^{*}$ Corresponding author: Shaheen Sultana

Assistant Professor, Department of Pharmaceutics, College of Pharmacy, Taif University, Postal Code: 21974, P.0.Box: 888. 
leading to end-stage renal disease (ESRD) or kidney failure [1]. ESRD is irreversible and fatal, unless treated by dialysis or kidney transplant.

CKD is a worldwide public health problem since the rapid increasing prevalence of relevant risk factors, like diabetes, hypertension, unhealthy diet, low to no physical activity and metabolic syndrome. It was a cause of 409,000 and 956,000 deaths in 1990 and 2013, respectively. Of those deaths, 46,000 (1990) and 173,000 (2013) were caused by CKD due to DM [2]. Recently, kidney diseases are the 12th cause of death and the 17th cause of disability at the global level, respectively [3]. CKD affects around $10-13 \%$ of the general population with an estimation of more than 500 million individual globally $[4,5]$. Recently, the Global Burden of Diseases, Injuries, and Risk Factors Study (GBD) ranked low glomerular filtration rate (GFR) as the 12th leading risk factor for death at the global level, and the 14th risk factor for Disability-Adjusted Life-Years (DALYs) among 79 risk factors in 2013 [3,6].

CKD is associated with eight- to ten- fold increased risk of kidney failure, cardiovascular disease (CVD), and premature death [4]. Other complications include acute kidney injury, increased risk of infection, cognitive decline, kidney failure, acute kidney injury, anemia, mineral and bone disorders, fractures and hospitalizations [7, 8]. However, early detection and treatment can prevent or delay the complications of CKD.

Major risk factors for the development and progression of CKD are diabetes and various cardiovascular diseases. CKD due to diabetes and hypertension affects nearly 5-7\% of the world population in developing countries [9]. Diabetes causes $9.1-29.9 \%$ of the cases of end stage renal disease (ESRD) in various developing countries, and hypertension contributes to $13-21 \%$ of the cases. Hypertension affects almost $25 \%$ of the adult population in Africa and is the cause of chronic kidney failure in $21 \%$ of patients on renal replacement therapy in South Africa. The prevalence of diabetic nephropathy is estimated to be $23.8 \%$ in Zambia, $14 \%-16 \%$ in South Africa, $12.4 \%$ in Egypt, 9\% in Sudan, $6.1 \%$ in Ethiopia, 43.2\% and 46.2\% in Taiwan and Hong Kong, respectively [10-12]. Previous studies also have demonstrated that the prevalence of CKD varies substantially among geographical regions which might be associated with the variability in diet pattern, lifestyle, education and economic condition $[13,14]$.

Chronic Kidney disease is also associated with huge economic burden which shares more than 2-3\% of their annual healthcare budget in high income countries, while patients with ESRD represent only 0.03\% of total population, and lower socioeconomic status is associated with greater risk of end-stage kidney disease [9]. In developing countries, the burden posed by CKD is much greater because of additional risks associated with poverty like: infections, hazardous work, poor education and poor maternal health combined with additional cost of screening and treatment where these costs have to be paid directly by patients $[15,16]$.

CKD is an important cause of death and disability worldwide. There are no studies in literature which determine the associated risk factors for CRD in Taif region, Saudi Arabia. Therefore, we will conduct a cross-sectional study to provide current data on the associated risk factors among CKD patients in Taif, Saudi Arabia. Accurate assessment of CKD risk factors among the general population and knowledge may provide important evidence-based information for policy makers and healthcare professionals regarding strategies for CKD prevention and healthcare planning. Screening for clinical indicators of renal dysfunction is influential for the early detection of patients at risk for CKD. This can also increase patients' awareness in order to modify their lifestyle towards preventing the occurrence of the disease [17].

Therefore, the current study is aimed at assessing risk factors and patient awareness for CKD in Taif region, Saudi Arabia.

\section{Methods}

We performed a case control study to assess the risk factors of CKD in patients from Alhada Military Hospital, and the general public awareness of the disease in Taif from September 2019 to March 2020. The study protocol was approved by the research and ethics committee of Taif University and Alhada Military hospital.

\subsection{Assessment of risk factors for chronic kidney diseases}

\subsection{Inclusion Criteria}

Patients aged $>18$ years old and suffering from kidney disease for 3 months or more.

\subsubsection{Exclusion Criteria}

(i) CKD patients who will be critically ill and unable to communicate in the study period will be excluded from the study. 
(ii) Pregnant women will be excluded from the study.

\subsubsection{Study Subjects}

The subjects were recruited from Al-Hada Military hospital in Taif from September 2019 to April 2020.

\subsubsection{Sampling procedure}

Case control study involving a face-to-face interview to answer a questionnaire (Appendix-I) for data collection by the researchers in the hospital. Cases were selected from the inpatient and outpatient settings of the nephrology department at Al-Hada Military hospital in Taif using simple random sampling technique. The controls that are nonCRD subjects were selected from the visitors and care givers accompanying the patients in the hospital. The criteria for selecting the controls were defined as the absence of renal damage (eGFR more than $60 \mathrm{~mL} / \mathrm{min} / 1.73 \mathrm{~m}^{2}$ or absence of albuminuria.)

\subsubsection{Study design}

Data was collected by face-to-face interview with the patients to answer the questionnaire (Appendix-I). All subjects completed a questionnaire recording their sociodemographic status (e.g., age, gender, education and personal income, etc.), personal and family health history (e.g., diabetes, hypertension, hepatitis, etc.), awareness and control of chronic non-communicable disease (e.g., diabetes, hypertension, dyslipidemia etc.), lifestyle (e.g., smoking, alcohol consumption, physical activity, etc.) and dietary pattern with the assistance of medical students, trained practitioners, doctors and nurses. Education was classified into the following three categories: uneducated, <12th grade and secondary school graduated or more. Current income status was categorized as difficult all the time, not too bad/good or good. Anthropometric measurements, such as height, weight and blood pressure (BP) was obtained from the patients' medical records and body mass index (BMI, $\mathrm{kg} / \mathrm{m}^{2}$ ) was calculated subsequently. BMI was divided into four categories: underweight $\left(<18.5 \mathrm{~kg} / \mathrm{m}^{2}\right)$, healthy weight $\left(18.5-24.9 \mathrm{~kg} / \mathrm{m}^{2}\right)$, overweight $\left(25-29.9 \mathrm{~kg} / \mathrm{m}^{2}\right)$ and obesity $\left(\geq 30 \mathrm{~kg} / \mathrm{m}^{2}\right)$. Hypertension was defined as participants whose average systolic blood pressure (SBP) $\geq 130 \mathrm{~mm} \mathrm{Hg}$, and/or average diastolic blood pressure (DBP) $\geq 80 \mathrm{~mm}$ Hg. The mean value of three blood pressure readings was used for statistical analysis, unless the difference between the readings was higher than $10 \mathrm{~mm} \mathrm{Hg}$, in which case the mean value of other two closest results was used. Participants who have practiced over 300 minutes of any regular physical activity per week were categorized as active, those who practiced 150-300 minutes of moderate physical activity per week (e.g. fast walking and swimming) were categorized as moderately active, and those who practiced less than 150 minutes of any physical activity per week were categorized as inactive including those with sedentary lifestyle $[2,6]$.

Daily consumption of fruits and vegetables was categorized into: the consumption of fewer than 2 fruits and vegetables per day was categorized as 'low', the consumption of 3 to 5 fruits and vegetables per day was categorized as 'medium, the consumption of more than 5 fruits and vegetables per day was categorized as 'high'. To assess the smoking status participants were grouped into: 'daily smoker', 'occasional smoker' and 'non-smoker'. An occasional smoker was someone who is not a daily smoker or has smoked less than 100 cigarettes in their lifetime while non-smokers were those who never smoke $[13,14]$.

\subsection{Assessment of public knowledge of chronic kidney disease}

The initial draft of the CKD knowledge assessment questionnaire was generated through literature review of existing questionnaires [18-20] (Appendix-II). The questionnaire included a total of ten evidence-based questions on the physiology of the kidneys, risk factors for CKD and signs and symptoms of CKD. The questionnaire was reviewed nephrologists $(n=4)$, renal nurses $(n=4)$ and research personnel $(n=4)$ for content validity and appropriateness. The reviewers were asked to evaluate all items of the questionnaire thoroughly and focusing the one that were deemed inappropriate in terms of phrasing and applicability. Consequently, items that would require a clinical level of expertise were deleted, and rephrasing of others which were not found to be appropriate in reviewer's opinion.

Eligible people from the general population to answer the questionnaire for assessing their basic knowledge of CKD were adults ( $\geq 18$ years old), not a registered healthcare professional, such as a doctor, nurse, pharmacist or dietitian, and did not have a personal history of kidney failure. Eligible participants were asked to answer an online-based questionnaire to assess their knowledge of CKD. Furthermore, to determine the discriminant validity of the questionnaire, two additional groups of participants were recruited: final (sixth) year undergraduate pharmacy students and nephrologists. Pharmacy students from Taif University will be invited to answer the online questionnaire during their regular university tutorial sessions. Nephrologists from AL-Hada Military hospital were selected to answer the questionnaire. Informed consent was obtained from all participants. 


\subsection{Statistical analysis}

All statistical analyses will be conduct using SPSS software version 19.0 (SPSS, Chicago, IL, USA). At a confidence interval of $95 \%$, a $\mathrm{P}$ value $<0.050$ was considered the significance threshold. Data was expressed as mean \pm SD, median with rang or frequency with percentages, as appropriate. To assess the risk factor, the risk of the outcome in the exposed group was compared with that in the non-exposed group by calculating relative risk ratio (RR) and odd ratio (OR) with its $95 \%$ CI using $2 \times 2$ contingency table. Intergroup comparisons were made using Pearson Chi-square test to examine statistical significant difference between the groups [13].

\section{Results}

\subsection{Assessment of risk factors contribute to CKD}

\subsubsection{Socio-demographic characteristics}

The study included 456 CKD patients and 600 controls (non-CKD patients). Table 1 presents their demographic characteristics. The mean age of the study participants was $55.21 \pm 12.47$ years. Among the total study population, $99 \%$ of participants were CKD stage III-V patients (Figure 1). The majority, $n=372(81.57 \%)$, of the participants were $>50$ years old $\left(\chi^{2}\right.$ value $\left.=5.21\right)$, male $\left[n=244(53.5 \%),\left(\chi^{2}\right.\right.$ value $\left.\left.=2.60\right)\right]$, married $\left[n=400(87.71 \%),\left(\chi^{2}\right.\right.$ value $\left.\left.=0.0115\right)\right]$ and uneducated $\left[\mathrm{n}=184(40.35 \%),\left(\chi^{2}\right.\right.$ value $\left.\left.=88.23\right)\right] .57 .01 \%$ of the participants had enough income; while $10.53 \%$ and $32.46 \%$ revealed difficult income status and not too bad/good in the CKD, respectively. Whereas in the control group $66 \%$ were secondary school graduates and $75.5 \%$ had good income status.

Table 1 Assessment of Sociodemographic Characteristics contribute to CKD.

\begin{tabular}{|c|c|c|c|c|}
\hline Sociodemographic Characteristics & With CKD *N (\%) & Without CKD **N (\%) & $\chi^{2}$ value & $p$ value \\
\hline Age (Average $\pm S D$ ) & $55.21 \pm 12.47$ & $54.26 \pm 16.22$ & - & 0.299 \\
\hline \multicolumn{5}{|l|}{ Age } \\
\hline $\begin{array}{l}18-24 \\
25-50 \\
>50\end{array}$ & $\begin{array}{l}16(3.5 \%) \\
68(14.91 \%) \\
372(81.57 \%)\end{array}$ & $\begin{array}{l}40(6.67 \%) \\
90(15 \%) \\
470(78.33 \%)\end{array}$ & 5.21 & 0.07 \\
\hline \multicolumn{5}{|l|}{ Time period of CKD } \\
\hline (Years \pm S.D) & $4.8 \pm 4.86$ & - & - & \\
\hline \multicolumn{5}{|l|}{ Gender } \\
\hline $\begin{array}{l}\text { Male } \\
\text { Female }\end{array}$ & $\begin{array}{l}244(53.5 \%) \\
212(46.49 \%)\end{array}$ & $\begin{array}{l}291(48.5 \%) \\
309(53.5 \%)\end{array}$ & 2.60 & 0.10 \\
\hline \multicolumn{5}{|l|}{ Marital Status } \\
\hline $\begin{array}{l}\text { Single } \\
\text { Married }\end{array}$ & $\begin{array}{l}56(12.28 \%) \\
400(87.71 \%)\end{array}$ & $\begin{array}{l}75(12.5 \%) \\
525(87.5 \%)\end{array}$ & 0.0115 & 0.915 \\
\hline \multicolumn{5}{|l|}{ Educational attainment } \\
\hline $\begin{array}{l}\text { Uneducated } \\
\text { 12th or less } \\
\text { Secondary school/graduated or higher }\end{array}$ & $\begin{array}{l}184(40.35 \%) \\
180(39.7 \%) \\
92(20.17 \%)\end{array}$ & $\begin{array}{l}24(4 \%) \\
180(30 \%) \\
396(66 \%)\end{array}$ & 88.63 & $<0.00001$ \\
\hline \multicolumn{5}{|l|}{ Income management } \\
\hline $\begin{array}{l}\text { Impossible/difficult all the time } \\
\text { Not too bad } \\
\text { Good }\end{array}$ & $\begin{array}{l}48(10.53 \%) \\
148(32.46 \%) \\
260(57.01 \%)\end{array}$ & $\begin{array}{l}30(5 \%) \\
117(19.5 \%) \\
453(75.5 \%)\end{array}$ & 11.80 & 0.00273 \\
\hline
\end{tabular}




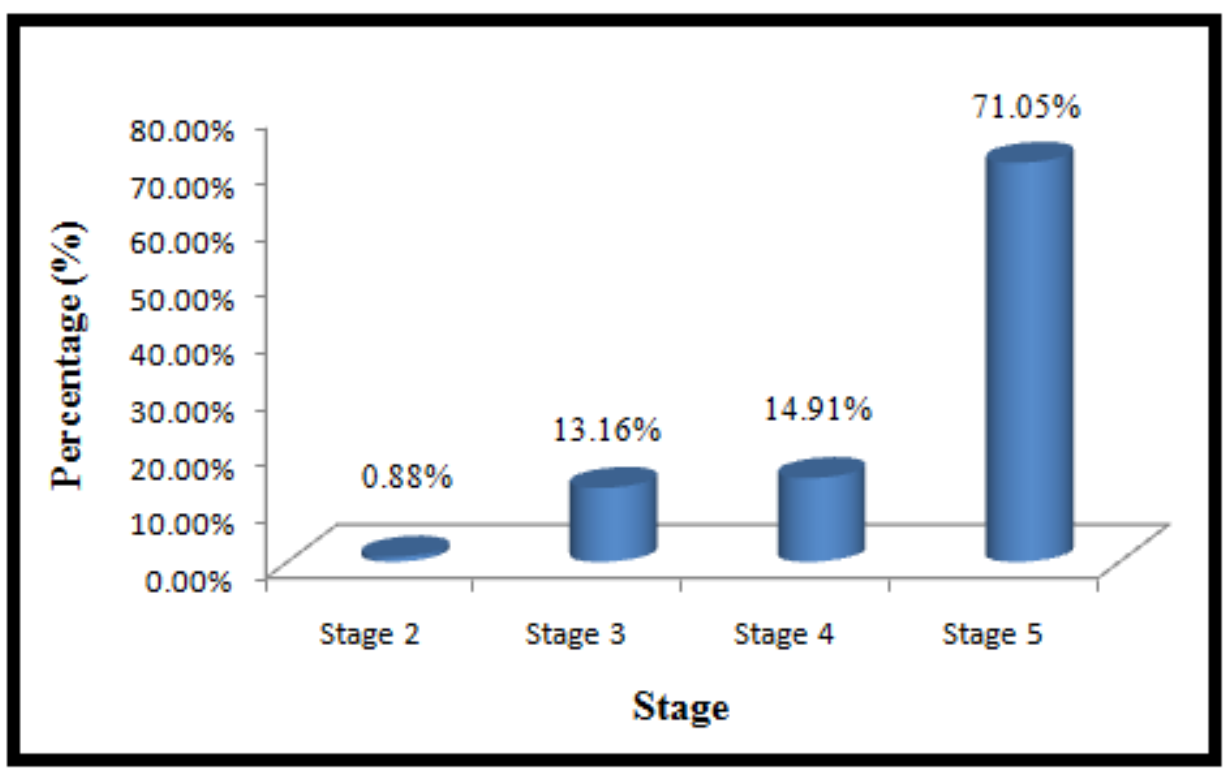

Figure 1Percent of participants with stage of CKD

\subsubsection{Biomedical Factors}

The results of the assessment of biomedical factors contribute to CKD is shown in Table 2. Among the study participants, 71.93\% [RR= $2.32(\mathrm{CI}=2.03-2.65), \mathrm{OR}=5.70(\mathrm{CI}=(4.36-7.45)]$ had type $2 \mathrm{DM}, 86.84 \%(\mathrm{RR}=9.65(\mathrm{CI}=7.46-12.48), 0 \mathrm{R}=$ 66.73 (CI=(45.19-98.54)]had hypertension and 57.89\% [RR=3.62 ( $\mathrm{CI}=2.96-4.42), \mathrm{OR}=7.22(\mathrm{CI}=(5.42-9.62)]$ had a history of heart diseases. Family history of CKD was found in $42.10 \%$ participants with risk ratio of 2.63 (CI= 2.13-3.26) and Odd ratio of 3.82 (CI= 2.87-5.09). 28.07\%, 42.10\% and $41.23 \%$ of the participants had significant high blood triglycerides level $\left(\chi^{2}\right.$ value $\left.=21.12\right)$, LDL level $\left(\chi^{2}\right.$ value $\left.=31.26\right)$ and uric acid levels $\left(\chi^{2}\right.$ value $\left.=54.35\right)$ when compared with the control group $(\mathrm{p}<0.00001)$. More than half of the subjects $(63.15 \%)$ in CKD stages III-V were overweight $(42.98 \%)$ or obese $(20.17 \%) .33 .33 \%$ participants had past kidney stone history $\left(\chi^{2}\right.$ value $\left.=52.63, p<0.05\right)$ and $71.93 \%$ of participants were anemic. $67.54 \%$ subjects had low vitamin D level, whereas calcium level was found to be a nonsignificant contributing factor to CKD.

Table 2Assessment of Biomedical factors contribute to CKD.

\begin{tabular}{|c|c|c|c|c|c|c|}
\hline $\begin{array}{l}\text { Biomedical } \\
\text { Factors }\end{array}$ & $\begin{array}{l}\text { With CKD } \\
\mathrm{N}^{*}(\%)\end{array}$ & $\begin{array}{l}\text { Without } \quad \text { CKD } \\
\mathrm{N}^{*}(\%)\end{array}$ & $\chi^{2}$ value & $P$ value & Risk ratio & Odd ratio \\
\hline \multicolumn{7}{|l|}{$\mathrm{BMI} * *$} \\
\hline $\begin{array}{l}\text { Underweight } \\
\text { Healthy weight } \\
\text { Overweight } \\
\text { Obese }\end{array}$ & $\begin{array}{l}8(1.75 \%) \\
60(35.09 \%) \\
96(42.98 \%) \\
92(20.17 \%)\end{array}$ & $\begin{array}{l}120(20 \%) \\
309(51.5 \%) \\
153(25.5 \%) \\
18(3 \%)\end{array}$ & 52.53 & $\begin{array}{l}< \\
0.00001\end{array}$ & $\begin{array}{l}2.21 \\
2.56) \quad(1.91-\end{array}$ & $4.30(3.31-5.58)$ \\
\hline \multicolumn{7}{|c|}{ Family History of CKD } \\
\hline $\begin{array}{l}\text { Yes } \\
\text { No }\end{array}$ & $\begin{array}{l}192(42.10 \%) \\
264(57.89 \%)\end{array}$ & $\begin{array}{l}96(16 \%) \\
504(84 \%)\end{array}$ & 26.06 & $\begin{array}{l}< \\
0.00001\end{array}$ & $\begin{array}{l}2.63 \quad(2.13- \\
3.26)\end{array}$ & $3.82(2.87-5.09)$ \\
\hline \multicolumn{7}{|c|}{ Personal History of Hypertension $* * *$} \\
\hline $\begin{array}{l}\text { Yes } \\
\text { No }\end{array}$ & $\begin{array}{l}396(86.84 \%) \\
60(13.15 \%)\end{array}$ & $\begin{array}{l}54(9 \%) \\
546(91 \%)\end{array}$ & 188.21 & $\begin{array}{l}< \\
0.00001\end{array}$ & $\begin{array}{l}9.65 \\
12.48)\end{array}$ & $\begin{array}{l}66.73 \\
98.54)\end{array}$ \\
\hline \multicolumn{7}{|c|}{ Personal History of Diabetes } \\
\hline $\begin{array}{l}\text { Yes } \\
\text { No }\end{array}$ & $\begin{array}{l}328(71.93 \%) \\
128(28.07 \%)\end{array}$ & $\begin{array}{l}186(31 \%) \\
414(69 \%)\end{array}$ & 48.99 & $\begin{array}{l}< \\
0.00001\end{array}$ & $\begin{array}{l}2.32 \\
2.65) \quad(2.03-\end{array}$ & $5.7(4.36-7.45)$ \\
\hline
\end{tabular}




\begin{tabular}{|c|c|c|c|c|c|c|c|}
\hline \multicolumn{7}{|c|}{ History of heart disease or stroke } & \multirow[b]{2}{*}{$7.22(5.42-9.62)$} \\
\hline $\begin{array}{l}\text { Yes } \\
\text { No }\end{array}$ & $\begin{array}{l}264(57.89 \%) \\
192(42.10 \%)\end{array}$ & $\begin{array}{l}96(16 \%) \\
504(84 \%)\end{array}$ & 59.36 & $\begin{array}{l}< \\
0.00001\end{array}$ & $\begin{array}{l}3.62 \\
4.42)\end{array}$ & $(2.96-$ & \\
\hline \multicolumn{7}{|c|}{ Blood triglyceride levels } & \\
\hline $\begin{array}{l}\text { Normal } \\
\text { High }\end{array}$ & $\begin{array}{l}328(71.93 \%) \\
128(28.07 \%)\end{array}$ & $\begin{array}{l}549(91.5 \%) \\
51(8.5 \%)\end{array}$ & 21.12 & $\begin{array}{l}< \\
0.00001\end{array}$ & $\begin{array}{l}3.3 \\
4.46)\end{array}$ & $(2.44-$ & $4.2(2.95-5.97)$ \\
\hline \multicolumn{7}{|c|}{ Total LDL } & \\
\hline $\begin{array}{l}\text { Normal } \\
\text { High }\end{array}$ & $\begin{array}{l}264(57.89 \%) \\
192(42.10 \%)\end{array}$ & $\begin{array}{l}516(86 \%) \\
84(14 \%)\end{array}$ & 31.26 & $\begin{array}{l}< \\
0.00001\end{array}$ & $\begin{array}{l}3.01 \\
4.46)\end{array}$ & $(2.44-$ & $4.47(2.95-5.97)$ \\
\hline \multicolumn{8}{|c|}{ Uric Acid } \\
\hline $\begin{array}{l}\text { Normal } \\
\text { High }\end{array}$ & $\begin{array}{l}268(58.77 \%) \\
188(41.23 \%)\end{array}$ & $\begin{array}{l}558(93 \%) \\
42(7 \%)\end{array}$ & 54.35 & $\begin{array}{l}< \\
0.00001\end{array}$ & $\begin{array}{l}5.89 \\
8.04)\end{array}$ & $(4.31-$ & $\begin{array}{l}9.32 \\
13.42)\end{array}$ \\
\hline \multicolumn{8}{|l|}{ Anemia } \\
\hline $\begin{array}{l}\text { Yes } \\
\text { No }\end{array}$ & $\begin{array}{l}328(71.93 \%) \\
128(28.07 \%)\end{array}$ & $\begin{array}{l}201(33.5 \%) \\
399(66.5 \%)\end{array}$ & 43.0062 & $\begin{array}{l}< \\
0.00001\end{array}$ & $\begin{array}{l}2.15 \\
2.44)\end{array}$ & $(1.89-$ & $5.09(3.90-6.63)$ \\
\hline \multicolumn{8}{|c|}{ Kidney stone } \\
\hline $\begin{array}{l}\text { Present } \\
\text { Yes } \\
\text { No } \\
\text { Past } \\
\text { Yes } \\
\text { No }\end{array}$ & $\begin{array}{l}56(12.28 \%) \\
400 \\
(87.71 \%) \\
152(33.33 \%) \\
304(66.67 \%)\end{array}$ & $\begin{array}{l}45(7.5 \%) \\
555(92.5 \%) \\
21(3.5 \%) \\
579(96.5 \%)\end{array}$ & 52.6385 & $\begin{array}{l}< \\
0.00001\end{array}$ & $\begin{array}{l}1.64 \\
2.38) \\
\\
9.52 \\
14.78)\end{array}$ & $\begin{array}{l}\text { (1.12- } \\
\text { (6.13- }\end{array}$ & $\begin{array}{l}1.73(1.14-2.61) \\
13.79 \\
22.21)\end{array}$ \\
\hline \multicolumn{8}{|c|}{ Vitamin D Level } \\
\hline $\begin{array}{l}\text { Low } \\
\text { Normal }\end{array}$ & $\begin{array}{l}308(67.54 \%) \\
148(32.46)\end{array}$ & $\begin{array}{l}216(36 \%) \\
384(64 \%)\end{array}$ & 28.975 & $\begin{array}{l}< \\
0.00001\end{array}$ & $\begin{array}{l}1.88 \\
2.12)\end{array}$ & (1.67- & $3.7(2.86-4.79)$ \\
\hline \multicolumn{8}{|c|}{ Calcium level } \\
\hline $\begin{array}{l}\text { Low } \\
\text { Normal }\end{array}$ & $\begin{array}{l}216(47.37 \%) \\
240(52.63 \%)\end{array}$ & $\begin{array}{l}258(43 \%) \\
342(57 \%)\end{array}$ & 0.56 & 0.45 & $\begin{array}{l}1.1 \\
1.26)\end{array}$ & $(0.96-$ & $1.19(0.93-1.52)$ \\
\hline
\end{tabular}

\subsubsection{Lifestyle Factors}

The results of assessment of lifestyle factors contribute to CKD are shown in Table 3. Physical activity $((\chi 2$ value $=$ 108.46) and smoking $((\chi 2$ value $=24.88)$ were significantly different between the two groups with $p$ values of $<0.00001$ and $<0.000016$, respectively. In CKD group, $28.07 \%$ subjects had low physical activity with risk ratio of 11.40 (CI=7.6117.09 ) and odd ratio of 20.13 (12.86-31.51). In contrast with the control group, only $4 \%$ had low physical activity level in their daily routine $(\mathrm{p}<0.00001)$. When considering smoking as a risk factor for CKD, $5 \%$ of the control group was confirmed as regular smokers while in the CKD group, $16.67 \%$ and $13.16 \%$ population was found to be regular and occasional smokers, respectively. 
Table 3 Assessment of lifestyle factors contribute to CKD.

\begin{tabular}{|c|c|c|c|c|c|c|}
\hline Lifestyle Factor & $\begin{array}{l}\text { With CKD } \\
{ }^{*} \mathrm{~N}(\%)\end{array}$ & $\begin{array}{l}\text { Without CKD } \\
{ }^{*} \mathrm{~N}(\%)\end{array}$ & $\chi^{2}$ value & $P$ value & Risk ratio & Odd ratio \\
\hline \multicolumn{7}{|l|}{ **Physical activity } \\
\hline $\begin{array}{l}\text { High } \\
\text { Average } \\
\text { None/Low }\end{array}$ & $\begin{array}{l}48(10.53 \%) \\
160(35.09 \%) \\
248(28.07 \%)\end{array}$ & $\begin{array}{l}204(34 \%) \\
372(62 \%) \\
24(4 \%)\end{array}$ & 108.46 & $<0.00001$ & $\begin{array}{l}11.40 \\
17.09)\end{array}$ & $\begin{array}{l}20.13(12.86- \\
31.51)\end{array}$ \\
\hline \multicolumn{7}{|l|}{ ***Smoking } \\
\hline $\begin{array}{l}\text { Regular } \\
\text { Occasional } \\
\text { None } \\
\text { In past }\end{array}$ & $\begin{array}{l}76(16.67 \%) \\
60(13.16 \%) \\
304(66.67 \%) \\
16(3.5 \%)\end{array}$ & $\begin{array}{l}30(5 \%) \\
27(4.5 \%) \\
537(89.5 \%) \\
6(1 \%)\end{array}$ & 24.88 & 0.000016 & $\begin{array}{l}3.22 \quad(2.43- \\
4.28)\end{array}$ & $\begin{array}{l}4.21 \\
5.92)\end{array}$ \\
\hline
\end{tabular}

**Patients who practiced $>300$ minutes of any regular physical activity per week were categorized as high, those who practiced $150-300$ minutes of moderate physical activity like fast walking and swimming per week were categorized as average, those who practiced less than 150 minutes per week of physical activity were categorized as none/low.

${ }^{* * *}$ Smoking was grouped as 'daily smoker', 'occasional smoker' and 'non-smoker'. Occasional smoker is someone who is not daily a smoker or has smoked less than 100 cigarettes in their lifetime while 'non-smokers were those who never smoke.

\subsubsection{Dietary Factors}

The assessment the effect of dietary factors contribute to CKD is shown in Table 4 . In the control group, $71.5 \%$ of the participants had an average daily fruits and vegetables intake, while 5.5\% and $23 \%\left(\chi^{2}\right.$ value $\left.=22.86, p=0.000011\right)$ had a high and low daily fruits and vegetables intake, respectively. In the CKD group, 37.71\% of the patients had low daily fruits and vegetables intake with risk ratio of $1.63(\mathrm{CI}=1.36-1.98)$ and odd ratio of $2(\mathrm{CI}=1.55-2.65)$, respectively. While $45.61 \%$ and $16.67 \%$ were found to have a diet average and high in fruits and vegetables. Similarly, in CKD groups, $28.07 \%$ and $29.82 \%$ had an average and high intake of dietary fats, respectively, compared with the control $\left(\chi^{2}\right.$ value= 96.66, $\mathrm{p}<0.00001$ ) where the percent for average and high fat intake is $83 \%$ and $10 \%$, respectively, which produces the risk ratio of 3.05( $\mathrm{CI}=2.31-4.03)$ and odd ratio of 3.92(CI=2.81-5.48). In case of fiber intake, 50.88\% and $42.10 \%$ of the CKD group had an average and low fiber intake, respectively. When compared to the control group $\left(\chi^{2}\right.$ value $=22.72, p=$ .000012 ), $71 \%$ and $17.5 \%$ had an average and low intake of dietary fiber, respectively. 58.5\% of the participants in the control group had an average meat intake compared to $30.70 \%$ in the CKD group. $20 \%$ and $47 \%$ of the control group and $37.72 \%$ and $42.10 \%$ of the CKD group take low and average sweets intake, respectively. Similarly, $7.5 \%$ and $49.5 \%$ take high and low salt intake $[\mathrm{RR}=1.22(\mathrm{CI}=1.09-1.35), \mathrm{OR}=1.56(\mathrm{CI}=1.22-2.0)]$, respectively, in the control group $\left(\chi^{2}\right.$ value $=9.45$ ), while $18.42 \%$ and $38.6 \%$ of the CKD group had a high and low salt intake, respectively. Soft drinks and water intake were considered insignificant risk factors.

Table 4 Assessment of dietary factors contribute to CKD.

\begin{tabular}{|c|c|c|c|c|c|c|}
\hline Dietary Factor & $\begin{array}{l}\text { With CKD } \\
{ }^{*} \mathrm{~N}(\%)\end{array}$ & $\begin{array}{l}\text { Without CKD *N } \\
\text { (\%) }\end{array}$ & $\chi^{2}$ value & $P$ value & Risk ratio & Odd ratio \\
\hline \multicolumn{7}{|c|}{ Fruits/Vegetables** } \\
\hline $\begin{array}{l}\text { High } \\
\text { Average } \\
\text { Low }\end{array}$ & $\begin{array}{l}76(16.67 \%) \\
208(45.61 \%) \\
172(37.71 \%)\end{array}$ & $\begin{array}{l}33(5.5 \%) \\
429(71.5 \%) \\
138(23 \%)\end{array}$ & 22.86 & .000011 & $\begin{array}{l}1.63 \\
1.98)\end{array}$ & $\begin{array}{l}2 \\
2.65)\end{array}$ \\
\hline \multicolumn{7}{|l|}{ Fat } \\
\hline $\begin{array}{l}\text { High } \\
\text { Average } \\
\text { Low }\end{array}$ & $\begin{array}{l}136(29.82 \%) \\
128(28.07 \%) \\
192(42.10 \%)\end{array}$ & $\begin{array}{l}60(10 \%) \\
498(83 \%) \\
56(7 \%)\end{array}$ & 96.66 & $<0.00001$ & $\begin{array}{l}3.05(2.31- \\
4.03)\end{array}$ & $\begin{array}{l}3.92(2.81- \\
5.48)\end{array}$ \\
\hline \multicolumn{7}{|l|}{ Fiber } \\
\hline
\end{tabular}




\begin{tabular}{|c|c|c|c|c|c|c|}
\hline $\begin{array}{l}\text { High } \\
\text { Average } \\
\text { Low }\end{array}$ & $\begin{array}{l}32(7.017 \%) \\
232(50.88 \%) \\
192(42.10 \%)\end{array}$ & $\begin{array}{l}69(11.5 \%) \\
426(71 \%) \\
105(17.5 \%)\end{array}$ & 22.72 & .000012 & $\begin{array}{l}2.40 \\
2.95)\end{array}$ & $\begin{array}{l}3.43 \\
4.54)\end{array}$ \\
\hline \multicolumn{7}{|l|}{ Red Meat } \\
\hline $\begin{array}{l}\text { High } \\
\text { Average } \\
\text { Low }\end{array}$ & $\begin{array}{l}96(21.05 \%) \\
140(30.70 \%) \\
220(48.24 \%)\end{array}$ & $\begin{array}{l}84(14 \%) \\
351(58.5 \%) \\
165(27.5 \%)\end{array}$ & 22.69 & .000012 & $\begin{array}{l}0.71 \\
0.79)\end{array}$ & $\begin{array}{l}0.41(0.31- \\
0.53)\end{array}$ \\
\hline \multicolumn{7}{|l|}{ Sweets } \\
\hline $\begin{array}{l}\text { High } \\
\text { Average } \\
\text { Low }\end{array}$ & $\begin{array}{l}92(20.17 \%) \\
192(42.10 \%) \\
172(37.72 \%)\end{array}$ & $\begin{array}{l}198(33 \%) \\
282(47 \%) \\
120(20 \%)\end{array}$ & 13.22 & .001345 & $\begin{array}{l}0.78(0.72- \\
0.84)\end{array}$ & $\begin{array}{l}0.41 \\
0.54)\end{array}$ \\
\hline \multicolumn{7}{|c|}{ Soft Drinks } \\
\hline $\begin{array}{l}\text { High } \\
\text { Average } \\
\text { Low }\end{array}$ & $\begin{array}{c}72(15.78 \%) \\
200(43.86 \%) \\
184(40.35 \%)\end{array}$ & $\begin{array}{l}96(16 \%) \\
231(38.5 \%) \\
273(45.5 \%) \\
\end{array}$ & 1.678 & 0.43 & $\begin{array}{l}1.09 \\
1.21)\end{array}$ & $\begin{array}{l}1.23 \\
1.58)\end{array}$ \\
\hline \multicolumn{7}{|l|}{ Water } \\
\hline $\begin{array}{l}\text { High } \\
\text { Average } \\
\text { Low }\end{array}$ & $\begin{array}{l}76(16.67 \%) \\
260(57.01 \%) \\
120(26.31 \%)\end{array}$ & $\begin{array}{l}138(23 \%) \\
285(47.5 \%) \\
177(29.5 \%) \\
\end{array}$ & 2.96 & 0.22 & $\begin{array}{l}0.71 \\
0.87)\end{array}$ & $\begin{array}{l}0.61(0.46- \\
0.81)\end{array}$ \\
\hline \multicolumn{7}{|l|}{ Salt } \\
\hline $\begin{array}{l}\text { High } \\
\text { Average } \\
\text { Low }\end{array}$ & $\begin{array}{l}84(18.42 \%) \\
196(42.98 \%) \\
176(38.6 \%)\end{array}$ & $\begin{array}{l}45(7.5 \%) \\
258(43 \%) \\
297(49.5 \%)\end{array}$ & 9.45 & .008874 & $\begin{array}{l}1.22 \\
1.35)\end{array}$ & $\begin{array}{l}1.56 \\
2.0)\end{array}$ \\
\hline
\end{tabular}

\subsubsection{Disease and medication related factors}

Results of assessment of disease and medication related factors contribute to CKD is shown in Table 5. CKD group has been associated with significant administration of several categories of drug including ACEI/ARBs, NSAIDs, antipsychotics, glucocorticoids, antidiabetics, statins, anticoagulants and diuretics when compared with control, which was $54.38 \%$ for ACEIs/ARBs [RR=2.098 ( $\mathrm{CI}=1.78-2.45), \mathrm{OR}=3.39$ (CI=2.62-4.40)], 16.67\% for NSAIDs [RR=5.33 ( $\mathrm{CI}=4.27-6.66), \mathrm{OR}=14$ ( $\mathrm{CI}=10.26-19.10)], 20.177 \%$ for antipsychotics [RR=2.69 (CI=1.92-3.76), OR=3.12 (2.13-4.56)], $34.21 \%$ for diuretics [RR=2.098 ( $\mathrm{CI}=1.78-2.45), \mathrm{OR}=14.34(\mathrm{CI}=8.90-23.09)], 51.75 \%$ for anti-diabetics [RR=2.46 ( $\mathrm{CI}=2.06-2.95), \mathrm{OR}=4.04$ ( $\mathrm{CI}=3.08-5.28)]$ and $52.63 \%$ for statins [RR=9.57 (CI=6.79-13.48), OR=19.09 (12.85-28.37)], respectively. The use of ACEIs/ARBs and NSAIDs in the CKD was significantly higher with the control. In the CKD group $20.17 \%$ of patients were taking antipsychotics when compared with control where only $7.5 \%$ of the control group was taking them. Similarly, intake of glucocorticoids, statins, antidiabetics, anticoagulants, and diuretics was significantly higher in the CKD group. $2.5 \%$ and $12.28 \%$ suffered from thyroid disorder in the control group and CKD group $(\chi 2$ value $=12.22$, $\mathrm{p}=.000473$ ), respectively. 
Table 5 Assessment of disease and medication related factors contribute to CKD.

\begin{tabular}{|c|c|c|c|c|c|c|}
\hline $\begin{array}{l}\text { Disease and } \\
\text { medication } \\
\text { related factors }\end{array}$ & $\begin{array}{l}\text { With CKD } \\
\text { *N (\%) }\end{array}$ & $\begin{array}{l}\text { Without } \\
\text { CKD } \\
* N(\%)\end{array}$ & $\chi^{2}$ value & $P$ value & Risk ratio & Odd ratio \\
\hline \multicolumn{7}{|l|}{ Medications } \\
\hline \multicolumn{7}{|l|}{ ACEIs/ARBs } \\
\hline $\begin{array}{l}\text { Yes } \\
\text { No }\end{array}$ & $\begin{array}{l}248(54.38 \%) \\
208(45.61 \%)\end{array}$ & $\begin{array}{l}156(26 \%) \\
444(74 \%)\end{array}$ & 25.301 & $<0.00001$ & $\begin{array}{l}2.098 \\
(1.78-2.45)\end{array}$ & $\begin{array}{l}3.39 \quad(2.62- \\
4.40)\end{array}$ \\
\hline \multicolumn{7}{|l|}{ NSAIDs } \\
\hline $\begin{array}{l}\text { Yes } \\
\text { No }\end{array}$ & $\begin{array}{l}304(66.67 \%) \\
152(33.33 \%)\end{array}$ & $\begin{array}{l}75(12.5 \%) \\
525(87.5 \%)\end{array}$ & 97.64 & $<0.00001$ & $\begin{array}{l}5.33 \\
6.66)\end{array}$ & $\begin{array}{l}14 \quad(10.26- \\
19.10)\end{array}$ \\
\hline \multicolumn{7}{|l|}{ Antibiotics } \\
\hline $\begin{array}{l}\text { Yes } \\
\text { No }\end{array}$ & $\begin{array}{l}100(21.93 \%) \\
356(78.07 \%)\end{array}$ & $\begin{array}{l}108(18 \%) \\
492(82 \%)\end{array}$ & 0.7164 & .397325 & $\begin{array}{l}1.22 \\
1.55)\end{array}$ & $\begin{array}{l}1.28 \\
1.73)\end{array}$ \\
\hline \multicolumn{7}{|l|}{ Antipsychotics } \\
\hline $\begin{array}{l}\text { Yes } \\
\text { No }\end{array}$ & $\begin{array}{l}92(20.17 \%) \\
364(79.82 \%)\end{array}$ & $\begin{array}{l}45(7.5 \%) \\
555(92.5 \%)\end{array}$ & 10.9673 & .000927 & $\begin{array}{l}2.69 \\
3.76)\end{array}$ & $\begin{array}{l}3.12 \text { (2.13- } \\
4.56)\end{array}$ \\
\hline \multicolumn{7}{|l|}{ Glucorticoids } \\
\hline $\begin{array}{l}\text { Yes } \\
\text { No }\end{array}$ & $\begin{array}{l}40(8.77 \%) \\
416(91.28 \%)\end{array}$ & $\begin{array}{l}12(2 \%) \\
588(98 \%)\end{array}$ & 7.817 & .005176 & $\begin{array}{l}4.39 \\
8.26)\end{array}$ & $\begin{array}{l}4.71 \quad(2.44- \\
9.09)\end{array}$ \\
\hline \multicolumn{7}{|l|}{ Antidiabetic } \\
\hline $\begin{array}{l}\text { Yes } \\
\text { No }\end{array}$ & $\begin{array}{l}236(51.75 \%) \\
220(48.24 \%)\end{array}$ & $\begin{array}{l}126(21 \%) \\
474(79 \%)\end{array}$ & 31.4759 & $<0.00001$ & $\begin{array}{l}2.46 \\
2.95)\end{array}$ & $\begin{array}{l}4.04 \\
5.28)\end{array}$ \\
\hline \multicolumn{7}{|l|}{ Statins } \\
\hline $\begin{array}{l}\text { Yes } \\
\text { No }\end{array}$ & $\begin{array}{l}240(52.63 \%) \\
216(47.37 \%)\end{array}$ & $\begin{array}{l}33(5.5 \%) \\
567(94.5 \%)\end{array}$ & 77.0572 & $<0.00001$ & $\begin{array}{l}9.57(6.79- \\
13.48)\end{array}$ & $\begin{array}{l}19.09 \\
(12.85- \\
28.37)\end{array}$ \\
\hline \multicolumn{7}{|l|}{ Anti-coagulant } \\
\hline $\begin{array}{l}\text { Yes } \\
\text { No }\end{array}$ & $\begin{array}{l}80(17.54 \%) \\
376(82.45 \%)\end{array}$ & $\begin{array}{l}18(3 \%) \\
582(97 \%)\end{array}$ & 20.2236 & $<0.00001$ & $\begin{array}{l}5.85 \\
9.61)\end{array}$ & $\begin{array}{l}6.88 \\
11.66)\end{array}$ \\
\hline \multicolumn{7}{|l|}{ Diuretic } \\
\hline $\begin{array}{l}\text { Yes } \\
\text { No }\end{array}$ & $\begin{array}{l}156(34.21 \%) \\
300(65.79 \%)\end{array}$ & $\begin{array}{l}21(3.5 \%) \\
579(96.5 \%)\end{array}$ & 54.7705 & $<0.00001$ & $\begin{array}{l}9.77 \\
15.16)\end{array}$ & $\begin{array}{l}14.34(8.90- \\
23.09)\end{array}$ \\
\hline \multicolumn{7}{|c|}{ Hormonal Imbalance } \\
\hline \multicolumn{7}{|l|}{ Cushing's syndrome } \\
\hline $\begin{array}{l}\text { Yes } \\
\text { No }\end{array}$ & $\begin{array}{l}0(0 \%) \\
456(100 \%)\end{array}$ & $\begin{array}{l}18(3 \%) \\
582(97 \%)\end{array}$ & 1.5012 & .220481 & 0 & 0 \\
\hline \multicolumn{7}{|l|}{ Sleep problems } \\
\hline $\begin{array}{l}\text { Yes } \\
\text { No } \\
\text { Sometimes }\end{array}$ & $\begin{array}{l}68(14.91 \%) \\
192(42.10 \%) \\
196(42.98 \%)\end{array}$ & $\begin{array}{l}96(16 \%) \\
270(45 \%) \\
234(39 \%)\end{array}$ & 0.4782 & .787326 & $\begin{array}{l}1.05 \\
1.17)\end{array}$ & $\begin{array}{l}1.13 \\
1.44)\end{array}$ \\
\hline
\end{tabular}




\begin{tabular}{|c|c|c|c|c|c|c|}
\hline \multicolumn{7}{|c|}{ Other: } \\
\hline \multicolumn{7}{|c|}{ Mental Disorder } \\
\hline $\begin{array}{l}\text { Yes } \\
\text { No }\end{array}$ & $\begin{array}{l}8(1.75 \%) \\
448(98.24 \%)\end{array}$ & $\begin{array}{l}3(0.5 \%) \\
597(99.5 \%)\end{array}$ & 1.2074 & .271851 & $\begin{array}{l}3.51(0.94- \\
13.15)\end{array}$ & $\begin{array}{l}3.55 \\
13.47)\end{array}$ \\
\hline \multicolumn{7}{|c|}{ Liver Diseases } \\
\hline $\begin{array}{l}\text { Yes } \\
\text { No }\end{array}$ & $\begin{array}{l}16(3.5 \%) \\
440(96.49 \%)\end{array}$ & $\begin{array}{l}48(8 \%) \\
552(92 \%)\end{array}$ & 2.4559 & .117081 & $\begin{array}{l}0.44 \quad(0.25- \\
0.76)\end{array}$ & $\begin{array}{l}0.42 \quad(0.23- \\
0.75)\end{array}$ \\
\hline \multicolumn{7}{|c|}{ Thyroid Disease } \\
\hline $\begin{array}{l}\text { Yes } \\
\text { No }\end{array}$ & $\begin{array}{l}56(12.28 \%) \\
400(87.72 \%)\end{array}$ & $\begin{array}{l}15(2.5 \%) \\
585(97.5 \%)\end{array}$ & 12.22 & .000473 & $\begin{array}{l}4.91 \text { (2.81- } \\
8.57)\end{array}$ & $\begin{array}{l}5.46 \\
9.79)\end{array}$ \\
\hline \multicolumn{7}{|c|}{ Other Diseases } \\
\hline $\begin{array}{l}\text { Yes } \\
\text { No }\end{array}$ & $\begin{array}{l}56(12.28 \%) \\
400(87.72 \%)\end{array}$ & $\begin{array}{l}63(10.5 \%) \\
537(89.5 \%)\end{array}$ & 0.2037 & .65174 & $\begin{array}{l}1.16(0.83- \\
1.64)\end{array}$ & $\begin{array}{l}1.19 \\
1.75)\end{array}$ \\
\hline
\end{tabular}

** Liver disorders included jaundice, hepatitis B, cirrhosis, hepatic impairment; mental disorders included dementia, anxiety, depression, socialphopia; thyroid disorder included hypothyroidism, carcinoma, and hyperthyroidism. Other diseases included latent TB, SLE, epilepsy, asthma, Posterior parathyroid adenoma, BPH, Pulmonary fibrosis, COPD, pulmonary edema and asthma

\subsection{Knowledge assessment of CKD of the general public}

A total of 2,119 people were invited to participate in the online survey. The survey was closed after three weeks, during which 1419 people accessed the survey and provided complete responses (response rate of 66.96\%).Table 6 shows participant characteristics.

Table 6 Baseline characteristics of different groups for knowledge assessment of CKD.

\begin{tabular}{|c|c|c|c|}
\hline \multirow[t]{2}{*}{ Characteristic } & \multicolumn{3}{|l|}{ n (\%) } \\
\hline & Public & Graduates & Nephrologist \\
\hline \multicolumn{4}{|l|}{ Age-Group years } \\
\hline $18-25$ & $713(50.24 \%)$ & $73(98.6 \%)$ & - \\
\hline $25-50$ & $590(41.58 \%)$ & $1(1.4 \%)$ & $10(100 \%)$ \\
\hline$>50$ & $116(8.17 \%)$ & $0(0 \%)$ & $0(0 \%)$ \\
\hline \multicolumn{4}{|l|}{ Gender } \\
\hline \multirow{2}{*}{$\begin{array}{l}\text { Male } \\
\text { Female }\end{array}$} & 567 (39.96\%) & $11(14.9 \%)$ & $10(100 \%)$ \\
\hline & $852(60 \%)$ & $63(85.1 \%)$ & $0(0 \%)$ \\
\hline \multicolumn{4}{|l|}{ Education } \\
\hline \multirow{3}{*}{$\begin{array}{l}\text { Less than high school } \\
\text { High school } \\
\text { College education }\end{array}$} & $58(4.09 \%)$ & $0(0 \%)$ & $0(0 \%)$ \\
\hline & $238(16.8 \%)$ & $0(0 \%)$ & $0(0 \%)$ \\
\hline & $1123(79.14 \%)$ & $100 \%$ & $100 \%$ \\
\hline \multicolumn{4}{|l|}{ Occupation } \\
\hline \multirow{4}{*}{$\begin{array}{l}\text { Students } \\
\text { Government employees } \\
\text { Self employed } \\
\text { Unemployed } \\
\end{array}$} & $566(39.88 \%)$ & $74(100 \%)$ & $0(0 \%)$ \\
\hline & $261(18.39 \%)$ & $0(0 \%)$ & $10(100 \%)$ \\
\hline & $66(4.565 \%)$ & $0(0 \%)$ & $0(0 \%)$ \\
\hline & $526(37 \%)$ & $0(0 \%)$ & $0(0 \%)$ \\
\hline
\end{tabular}

The majority of respondents were between the ages $18-50$ years old $(91.76 \%, n=1203)$ while 36 respondents were $>50$ years old (8.17\%). Majority of respondents were women $(60 \%, \mathrm{n}=852) .20 .89 \%(\mathrm{n}=296)$ of respondents had high school education or less while 79.14\% ( $n=1123)$ had college level education. Only 39.88\% $(n=566)$ of respondents were 
students, 4.565\% (n=66) were self-employed, 18.39\% $(n=261)$ were government employees, while $37 \%(n=526)$ were unemployed.

Table 7 shows the percentage of participants with corrects responses to individual items on the questionnaire. Most participants (79.14\%) knew that chronic kidney disease is a reduction in the kidneys' ability to remove wastes from the blood; however, many (41.1\%) have identified that blood in the urine can be a symptom of CKD while only $13.7 \% \mathrm{knew}$ that CKD can occur without any symptoms. Many participants (45.9\%) identified hypertension as a major risk factor when compared with diabetes (29.2\%), cardiovascular diseases (20.3\%) and obesity (24.4\%) all were less frequently recognized.

Most of the participants were familiar with the common symptoms of advanced chronic kidney diseases such as edema $(n=1045,73.7 \%)$, nausea/vomiting $(n=305,21.5 \%)$, loss of appetite $(n=285,20.1 \%)$ and increase fatigue ( $n=450$, $31.7 \%)$. Most of the population (63.7\%) knew that patients with hypertension, diabetes mellitus and family history of renal disease need to do screening on routine basis. 55.9\% $(\mathrm{n}=793)$ of the population revealed that patients with chronic kidney disease, hematuria and proteinuria must consult with a nephrologist. Only 44.3\% ( $n=629)$ and 45\% (n=639) knew that by controlling blood glucose and blood pressure, respectively, one can prevent chronic kidney disease; while the maximum $(n=1017,71.7 \%)$ had knowledge regarding healthy fluid intake as a preventive measure for CKD.

In comparison, $n=61(82.4 \%)$ graduates were familiar with CKD and knew that it is the reduced ability of the kidneys to remove wastes from the blood; only $48(64.9 \%)$ and $43(58.1 \%)$ of the respondents knew that hypertension and diabetes mellitus are risk factors for chronic kidney disease, respectively. While $60(81.08 \%)$ graduates were aware about the patients need to do screening, and $73 \%$ answered that a healthy fluid intake can be a preventive measure of CKD.

With all three groups, confusion was identified with symptom associated with CKD. 86.3\% and 50\% of participants in the general public and graduates, respectively, did not know that CKD may be entirely asymptomatic, especially in its early stages.

Table 7 Response of questionnaire by different groups.

\begin{tabular}{|c|c|c|c|c|}
\hline \multirow[t]{2}{*}{ Item } & \multirow[t]{2}{*}{ Question } & \multicolumn{3}{|c|}{ Responses n $(\%)^{* *}$} \\
\hline & & Public & Graduates & Nephrologist \\
\hline \multicolumn{5}{|c|}{ Q.1. What do you think CKD is? } \\
\hline 1 & An infection of the kidneys & $45(3.2 \%)$ & $6(8.1 \%)$ & - \\
\hline $2^{*}$ & $\begin{array}{l}\text { Reduction in kidneys' ability to } \\
\text { remove wastes from the blood }\end{array}$ & $\begin{array}{l}1123 \\
(79.14 \%)\end{array}$ & $61(82.4 \%)$ & $100 \%$ \\
\hline 3 & An inflammation of the kidney & $78(5.5 \%)$ & $4(5.4 \%)$ & - \\
\hline 4 & A stone of the kidneys & $38(2.7 \%)$ & $4(5.4 \%)$ & - \\
\hline 5 & Do not know/no response & $133(9.4 \%)$ & & - \\
\hline \multicolumn{5}{|c|}{ Q.2. How many healthy kidneys a person needs to lead a healthy life? } \\
\hline $6^{*}$ & One & $938(66.10 \%)$ & $46(62.2 \%)$ & $100 \%$ \\
\hline 7 & Two & $474(33.40 \%)$ & $27(36.5 \%)$ & - \\
\hline 8 & More than two & $7(0.5 \%)$ & & - \\
\hline \multicolumn{5}{|c|}{ Q.3. What is the function of a kidney? } \\
\hline 9 & To break down food & $20(1.4 \%)$ & $3(4.0 \%)$ & - \\
\hline 10 & $\begin{array}{l}\text { To produce substances that } \\
\text { break down fat }\end{array}$ & $55(3.9 \%)$ & $4(5.4 \%)$ & - \\
\hline $11^{*}$ & $\begin{array}{l}\text { To filter waste products in the } \\
\text { blood }\end{array}$ & $1247(87.9 \%)$ & $68(91.9 \%)$ & $100 \%$ \\
\hline
\end{tabular}




\begin{tabular}{|c|c|c|c|c|}
\hline 12 & Do not know & $97(6.8 \%)$ & - & - \\
\hline \multicolumn{5}{|c|}{ Q.4. What are the perceived cause(s) of chronic kidney diseases? } \\
\hline 13 & Inadequate sleep & $84(5.9 \%)$ & $2(2.7 \%)$ & \\
\hline 14 & Taking too much salt & $677(47.7 \%)$ & $25(33.8 \%)$ & $20 \%$ \\
\hline $15^{*}$ & High Blood pressure & $500(35.2 \%)$ & $48(64.9 \%)$ & $100 \%$ \\
\hline $16^{*}$ & Diabetes & $470(33.1 \%)$ & $48(64.9 \%)$ & $100 \%$ \\
\hline $17^{*}$ & Inherited condition & $402(28.3 \%)$ & $25(33.8 \%)$ & $80 \%$ \\
\hline 18 & Others & $467(32.9 \%)$ & $13(17.6 \%)$ & $40 \%$ \\
\hline \multicolumn{5}{|c|}{ Q.5. What is the most common symptoms of chronic kidney disease? } \\
\hline $19^{*}$ & Bubbles in the urine & $305(21.5 \%)$ & $47(63.6 \%)$ & $90 \%$ \\
\hline 20 & Frequent thirst & $220(15.5 \%)$ & $15(20.3 \%)$ & $10 \%$ \\
\hline 21 & Back pain & $485(34.2 \%)$ & $34(45.9 \%)$ & $80 \%$ \\
\hline $22^{*}$ & Blood in the urine & $583(41.1 \%)$ & $62(83.78 \%)$ & $100 \%$ \\
\hline 23 & Yellow eyes & $237(16.7 \%)$ & $12(16.2 \%)$ & \\
\hline $24^{*}$ & No symptoms or complaints & $194(13.7 \%)$ & $37(50 \%)$ & $80 \%$ \\
\hline 25 & Others & $311(21.9 \%)$ & $14(18.9 \%)$ & \\
\hline 26 & Do not Know & & $12(16.2 \%)$ & \\
\hline \multicolumn{5}{|c|}{ Q.6. What are the risk factors for chronic kidney disease? } \\
\hline $27^{*}$ & High blood pressure & $651(45.9 \%)$ & $48(64.9 \%)$ & $100 \%$ \\
\hline $28^{*}$ & Diabetes mellitus & $414(29.2 \%)$ & $43(58.1 \%)$ & $100 \%$ \\
\hline 29 & Female gender & $162(11.4 \%)$ & $2(2.7 \%)$ & \\
\hline $30^{*}$ & $\begin{array}{l}\text { Heart problems such as heart } \\
\text { failure or heart attack }\end{array}$ & $288(20.3 \%)$ & $35(47.3 \%)$ & $90 \%$ \\
\hline $31^{*}$ & Obesity & $346(24.4 \%)$ & $37(50 \%)$ & $60 \%$ \\
\hline 32 & Excessive stress & $182(12.8 \%)$ & $15(20.3 \%)$ & \\
\hline 33 & Other & $419(29.5 \%)$ & & \\
\hline \multicolumn{5}{|c|}{$\begin{array}{l}\text { Q.7. What are the signs and symptoms that a person might have if they have advanced chronic kidney disease } \\
\text { or kidney failure? }\end{array}$} \\
\hline $34^{*}$ & Water retention (edema) & $1045(73.7 \%)$ & $42(56.76 \%)$ & $9(90 \%)$ \\
\hline 35 & Fever & $220(15.5 \%)$ & $10(13.5 \%)$ & $1(10 \%)$ \\
\hline $36^{*}$ & Nausea/Vomiting & $305(21.5 \%)$ & $58(78.39 \%)$ & $10(100 \%)$ \\
\hline $37^{*}$ & Loss of appetite & $285(20.1 \%)$ & $60(81.08 \%)$ & $9(90 \%)$ \\
\hline $38^{*}$ & Increase fatigue (tiredness) & $450(31.7 \%)$ & $37(50 \%)$ & $10(100 \%)$ \\
\hline 39 & Do not Know & $275(19.4 \%)$ & $9(12.2 \%)$ & $0(0 \%)$ \\
\hline \multicolumn{5}{|c|}{ Q.8. Who need to do screening for (CKD)? } \\
\hline $40^{*}$ & Hypertension & $84(5.9 \%)$ & (3) $4.0 \%$ & $0(0 \%)$ \\
\hline $41^{*}$ & Diabetes mellitus & $53(3.7 \%)$ & (4) $5.4 \%$ & $0(0 \%)$ \\
\hline $42^{*}$ & $\begin{array}{l}\text { Patient with family history of } \\
\text { renal disease }\end{array}$ & $172(12.1 \%)$ & (3) $4.0 \%$ & $0(0 \%)$ \\
\hline
\end{tabular}




\begin{tabular}{|c|c|c|c|c|}
\hline $43^{*}$ & All of the above & $904(63.7 \%)$ & (60) $81.08 \%$ & $10(100 \%)$ \\
\hline 44 & None of the above & $33(2.3 \%)$ & $0(0 \%)$ & $0(0 \%)$ \\
\hline 45 & Do not Know & $159(11.2 \%)$ & $0(0 \%)$ & $0(0 \%)$ \\
\hline \multicolumn{5}{|c|}{ Q.9. Who need to be referred to nephrologist? } \\
\hline $46^{*}$ & $\begin{array}{l}\text { Patient with chronic kidney } \\
\text { staging } 3 \text { and above }\end{array}$ & $185(13 \%)$ & $8(10.6 \%)$ & $0(0 \%)$ \\
\hline $47^{*}$ & Proteinuria & $65(4.6 \%)$ & $4(5.3 \%)$ & $0(0 \%)$ \\
\hline $48^{*}$ & Haematuria & $104(7.3 \%)$ & $4(5.3 \%)$ & $0(0 \%)$ \\
\hline $49^{*}$ & All of them & $793(55.9 \%)$ & $52(70.24 \%)$ & $10(100 \%)$ \\
\hline 50 & None of the above & $10(0.7 \%)$ & $0(0 \%)$ & $0(0 \%)$ \\
\hline 51 & Do not Know & $263(18.5 \%)$ & $0(0 \%)$ & $0(0 \%)$ \\
\hline \multicolumn{5}{|c|}{ Q.10 Which of the following is recommended to prevent chronic kidney disease? } \\
\hline $52^{*}$ & Keep fit and active & $599(42.2 \%)$ & $20(27 \%)$ & $8(80 \%)$ \\
\hline $53^{*}$ & Keep blood glucose controlled & $629(44.3 \%)$ & $39(52.7 \%)$ & $10(100 \%)$ \\
\hline $54^{*}$ & Keep blood pressure controlled & $639(45 \%)$ & $43(58.1 \%)$ & (8) $80 \%$ \\
\hline 55 & Increase protein intake & $175(12.3 \%)$ & $7(9.5 \%)$ & $0(0 \%)$ \\
\hline $56^{*}$ & $\begin{array}{l}\text { Eat healthy and keep body } \\
\text { weight in check }\end{array}$ & $649(45.7 \%)$ & $36(48.64 \%)$ & $8(80 \%)$ \\
\hline $57^{*}$ & Maintain a healthy fluid intake & $1017(71.7 \%)$ & $54(73 \%)$ & $7(70 \%)$ \\
\hline
\end{tabular}

As shown in Figure 2 over $50 \%$ of the participants had knowledge scores less than 11 . The mean (SD) knowledge score of Taif general public was found to be $13.03( \pm 5.60)$ (Table 8). While the mean (SD) knowledge score for graduates and nephrologists was found to be 18.78( \pm 3.93$)$ and $24.7( \pm 2.23)$, respectively. The knowledge scores for different groups (public and graduates) were found to be statistically significant ( $\mathrm{p}<0.0001)$ when compared with nephrologists' (Table 4.8). The Kruskal-Wallis test revealed a statistically significant difference in the total score of participants across the three groups $(\chi 2(2, N=521)=86.598, p<0.001)$. Post-hoc comparisons were performed between pairs of groups found statistically significant differences between all three groups $(\mathrm{p}<0.001)$.

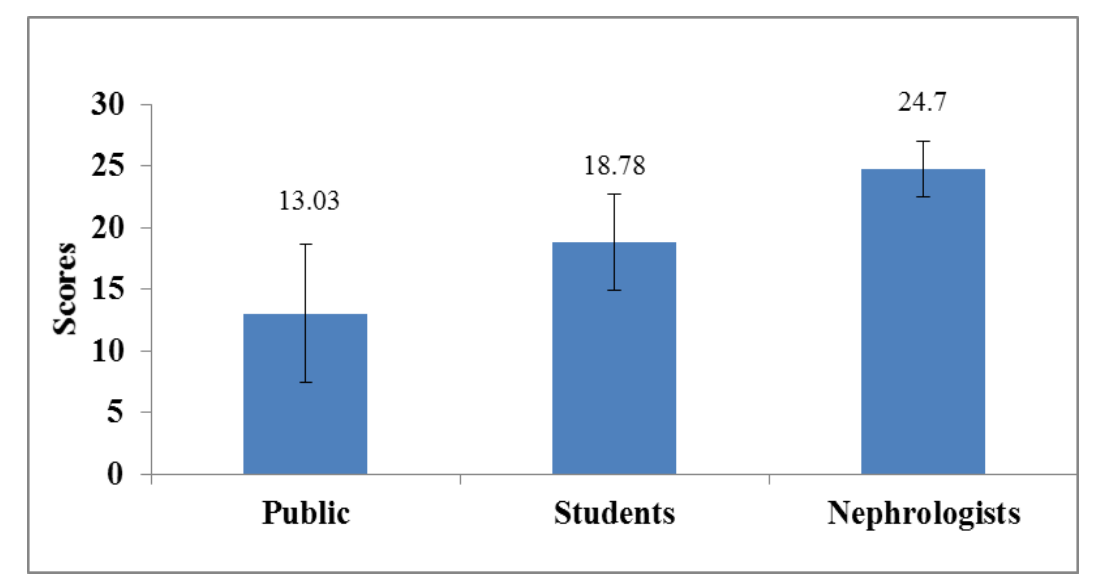

Figure 2 Mean (SD) knowledge score of different groups where the general public ( $\mathrm{n}=1419)$; graduates $(\mathrm{n}=74)$; nephrologists $(n=10)$ 
Table 8 Statistical results for three tested groups by Mann Whitney test

\begin{tabular}{|l|l|l|l|}
\hline Parameters & Public & Students & Nephrologist \\
\hline Mean \pm SD & $13.03 \pm 5.60$ & $18.78 \pm 3.93$ & $24.7 \pm 2.23$ \\
\hline Variance & 31.42 & 15.52 & 5.01 \\
\hline Standard Error & 0.2688 & 0.4611 & 0.2134 \\
\hline $95 \%$ CI & $12.505,13.555$ & $17.89,19.66$ & $23.318,26.082$ \\
\hline P value & $<0.0001$ & $<0.0001$ & \\
Difference & 11.60 & 5.920 & \\
Standard Error & 1.776 & 1.274 & Reference \\
$95 \%$ CI & $8.18,15.16$ & $3.38,8.45$ & \\
T statistics & 6.57 & 4.731 & \\
Df & 445 & 82 & \\
Z value & 5.143069 & 4.037372 & \\
\hline
\end{tabular}

A box plot design is showing in Figure 3 displaying variation in score among different groups. It can be clearly depicted that minimum variation was observed with nephrologists with score values ranging from 21 to 29; while the maximum variation can be seen with general public where the scores varies from 1 to 29 . On the other hand, average variation in the scores was observed with graduates where the value fluctuated between 10 to 26 .

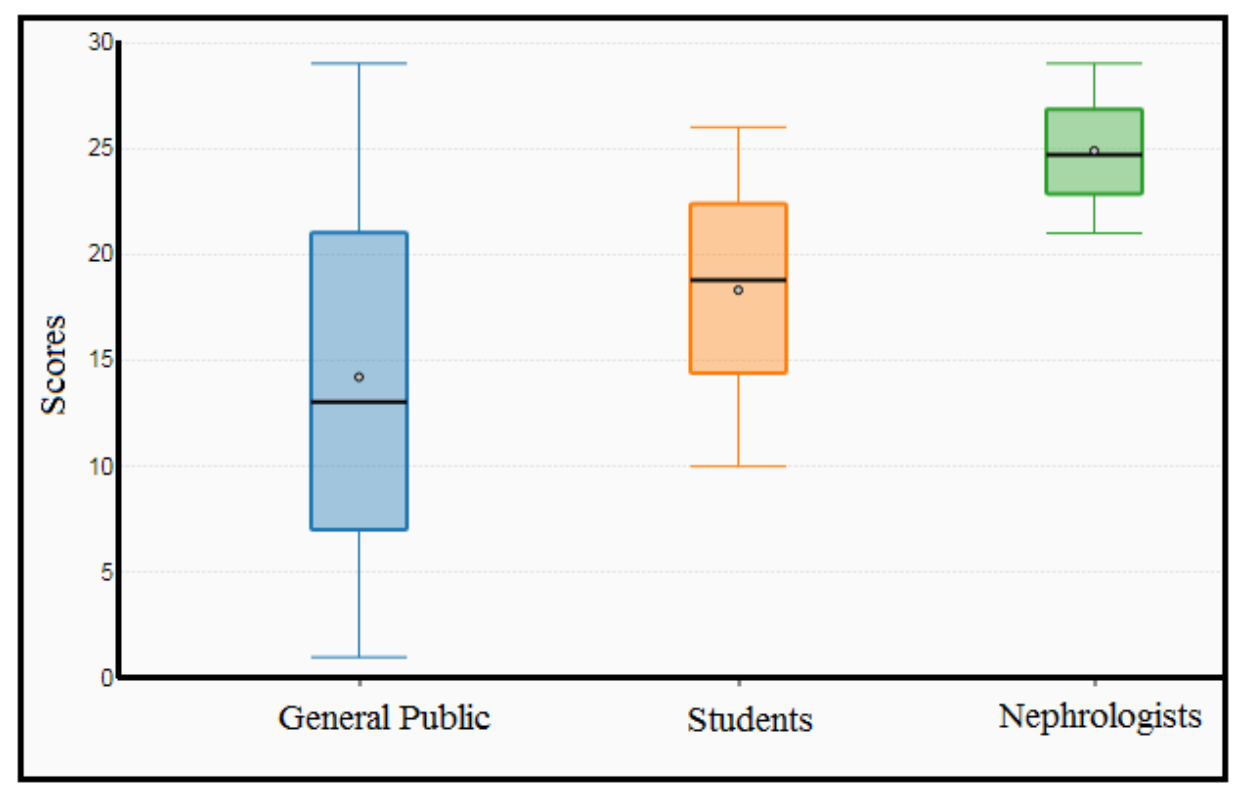

Figure 3 Box Plot showing mean knowledge scores of respondents; general public $(n=1419)$; graduates $(n=74)$; nephrologists $(\mathrm{n}=10)$

Distribution of scores as percentages for different groups is shown in Figure 4. For the general public the percentage of population acquired scores from 1 to 29 varied from $0.63 \%$ to $0.23 \%$, respectively. Highest percentage (9.15\%) got score of 11 , while the minimum percentage of respondent $(0.23 \%)$ got 28 and 29 scores. The percentage of graduate population acquired scores from 10 to 28 was $1.35 \%$. Highest percentage $(12.16 \%)$ of graduates got scores of 18 , while the minimum percentage of graduates (1.35\%) got 10,11, 27 and 28 scores. For nephrologist, the scores varied from 21 to 29 with $30 \%$ of respondent got a high score of 25 . 


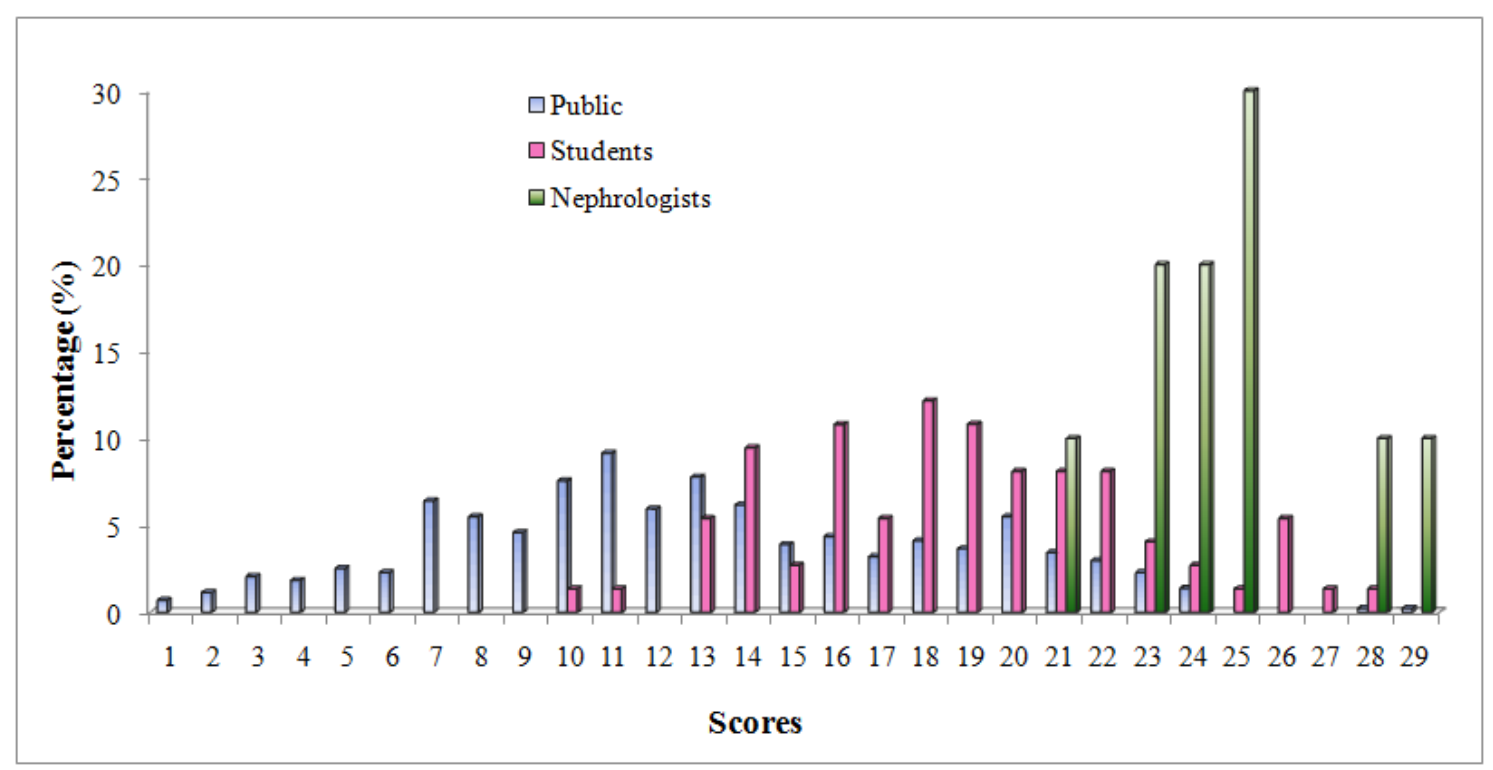

Figure 4 Distribution of the chronic kidney disease knowledge scores of the Public, Students and Nephrologists.

As shown in Figure 5 more than 50\% of the participants had knowledge scores less than 14. 919 (64.7\%) of the general public had poor knowledge, 273 (19.22\%) had fair knowledge, and 227 respondents (16.01\%) had good knowledge of CKD. In comparison, 13 graduates (17.56\%) had poor knowledge, 37 graduates (49.97\%) had fair knowledge and 24 graduates (32.2\%) had good knowledge of CKD. While, 100\% nephrologists had good knowledge scale.

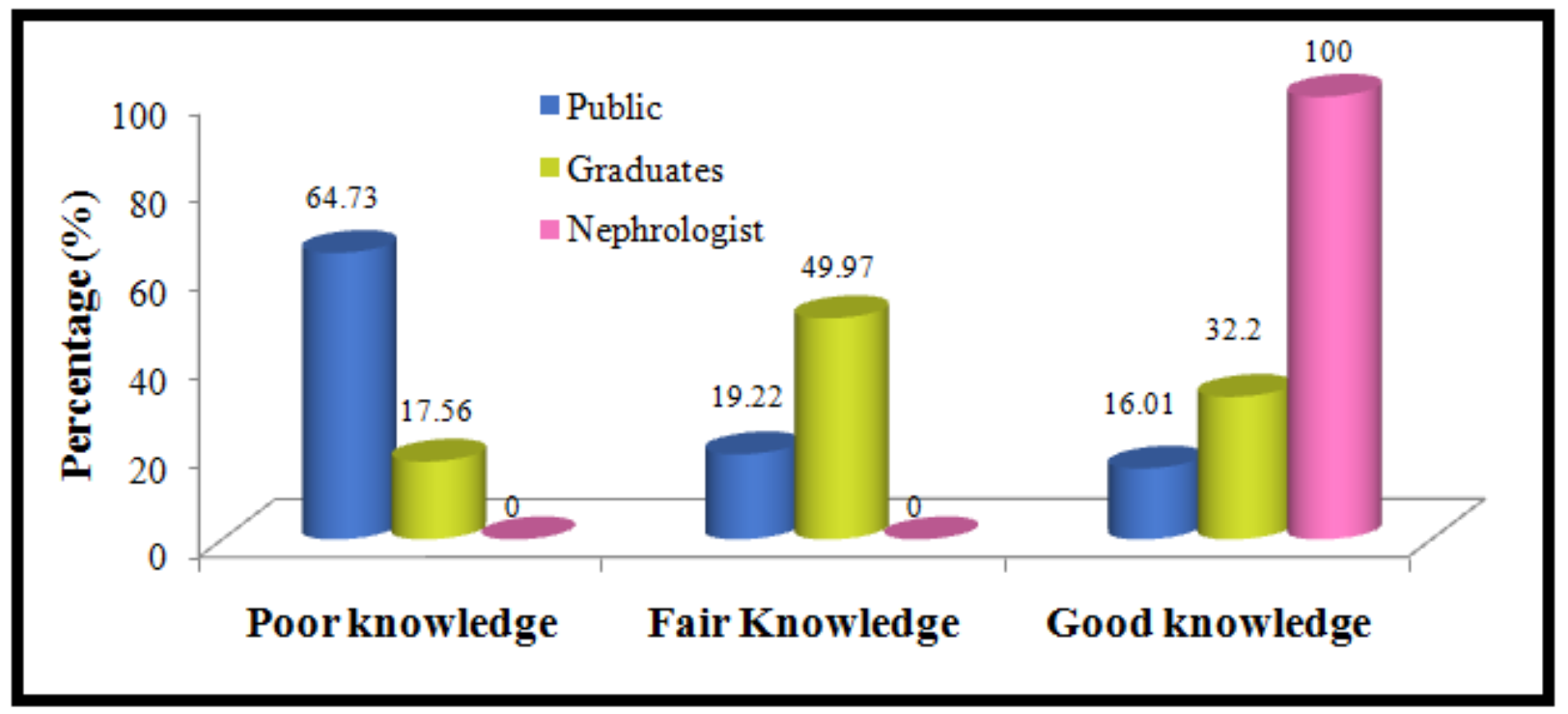

Figure 5 Knowledge of chronic kidney disease among respondents.

There was no statistically significant difference found among the age groups, gender and education of respondents on the knowledge of CKD (Table 9). However, we found a significant effect of occupation on knowledge score. Pair-wise comparison of group means with post hoc Turkey HSD test (honestly significant different) showed the mean knowledge score of government employees group was significantly higher than student groups $(\mathrm{p}=0.0129)$, and unemployed group ( $\mathrm{p}=0.0016)$. We found a non-significant difference between knowledge score and education level, but a higher knowledge score was found with college scale education level when compared with high school education or less than high school education. 
Table 9 Comparison of mean knowledge score of public regarding CKD amongst variables (demographic data)

\begin{tabular}{|l|l|l|l|l|}
\hline Variable & n (Percentage) & $\begin{array}{l}\text { Mean knowledge } \\
\text { score (SD) }\end{array}$ & F Statistics & P value \\
\hline Age & & & & \\
$18-25$ & $713(50.24 \%)$ & $13.35 \pm 5.56$ & 0.33 & 0.718 \\
$26-50$ & $590(41.58 \%)$ & $13.8 \pm 6.07$ & & \\
$>51$ & $116(8.17 \%)$ & $13.87 \pm 6.23$ & & \\
\hline Gender & & & 0.178 & 0.836 \\
Male & $567(39.96 \%)$ & $14.67 \pm 5.92$ & \\
Female & $852(60 \%)$ & $13.85 \pm 7.89$ & & \\
\hline Education & & & 0.797 \\
College Education & $58(4.09 \%)$ & $16.11 \pm 43.56$ & \\
High School education & $238(16.8 \%)$ & $13.34 \pm 5.88$ & \\
$<$ High school & $1123(79.14 \%)$ & $10.72 \pm 4.94$ & & \\
\hline Occupation & & & 3.53 & \\
Student & $566(39.88 \%)$ & $13.3 \pm 5.57$ & & \\
Government employees & $261(18.39 \%)$ & $15.24 \pm 6.16$ & & \\
Self Employed & $66(4.56 \%)$ & $13.15 \pm 6.42$ & & \\
Unemployed & $526(37 \%)$ & $12.73 \pm 5.56$ & & \\
\hline
\end{tabular}

Pair-wise comparison of group means with post hoc Turkey HSD (honestly significant different test showed a significant difference between students, government employees, self-employed and unemployed groups respectively.

\section{Discussion}

Chronic kidney disease (CKD) has been a global public health issue in the past decades and the development of CKD prevention and control strategies is an important decision for reducing the burden of the disease. Identifying individual's risk factors and at-risk populations are potential targets for a suitable intervention in different populations. This should include critical analysis of the existing social, biomedical, lifestyle and dietary risk factors at both the individual and the community level. In the present study the exposure to different risk factors of CKD is collectively very high, which requires more attention. In our study we assessed risk factors contribute to CKD in association with public awareness for CKD [21].

Previous study showed that CKD prevalence was associated with several indicators of SES, particularly lower income; lower education and lower combined SES [22]. Researchers reported that Low income $(<\$ 16,000 /$ year compared with income $>\$ 35,000 /$ year) was associated with higher (2.4-fold) increase in CKD [23]. We also find a close relationship between age, marital status, education level, income management and CKD.

Previous studies reported that a family history of CKD was a significant risk factor for end-stage renal disease (ESRD) [24]. We found a significant association between CKD and positive family history which supports the hypothesis. Such positive correlation was also reported by other researchers [25, 26]. Despite this significant association such relation had no impact on higher all-cause mortality rates than those without such a family history of CKD [58]. Screening of these high-risk relatives for early nephropathy, and for risk factors for nephropathy, will probably lead to successful reduction and slow the growing worldwide epidemic of CKD.

An elevated percentage of CKD was observed in hypertensive (86.84\%), heart disease or stroke (57.89\%), diabetic (71.93\%), overweight or obese (63.15\%) participants in our study. The likelihood for CKD significantly and independently increased by 3-fold and 4-fold in the presence of higher BMI [27-30], hypertension [31, 32], cardiovascular diseases [33] and diabetes [31,32]. Because of the well-known interactions between CVD, hypertension, diabetes, and CKD, these findings have important clinical and public-health implications; in targeting these "high-risk" population for ESRD we can reduce the progression and delay the onset of ESRD. In our study, elevated uric acid 
conferred a greater risk for CKD, which is consistent with other epidemiological studies demonstrating that uric acid is a strong and independent risk factor for renal disease [34]. Similarly, our findings suggest a positive correlation between history of kidney stones and an increased risk estimate for CKD. A similar finding suggested that female patients with kidney stones showed a slightly higher risk for CKD than male patients [35].

The accumulation of healthy lifestyle behaviors has shown to be associated with the prevention of several diseases such as CVD, stroke, type $2 \mathrm{DM}$, hypertension and dyslipidemia. Several reports clarified the association of CKD and healthy lifestyle behaviors that may help to demonstrate the importance of lifestyle modification in CKD prevention [36, 37]. Recently, cross-sectional study reported that unhealthy lifestyle behaviors, especially those related to lack of habitual moderate exercise, late-night dinner and bedtime snacking may be independent risk factors for the development of CKD [38]. Our results also speculate the same findings which further emphasize an accumulation of healthy lifestyle behaviors for prevention of CKD. High physical activity as well as non-smoking behavior had influence on the progression of CKD and may directly contribute to the prevention of the development of future ESRD or the introduction of dialysis in middle-aged and older males.

Healthy dietary patterns were consistently associated with lower mortality and thus could be an efficient tool to reduce mortality in people with kidney disease. We observed a significant correlation between CKD risk and intakes of dietary fruits and vegetables. CKD group was associated with lower intake of fruits, vegetables, fiber and high fat. A crosssectional study found that a dietary pattern including high intakes of whole grains, fruit, vegetables, and low-fat dairy foods was inversely associated with urinary albumin-to-creatinine ratio and the odds of microalbuminuria among adults without CVD, diabetes or microalbuminuria [39]. While our study revealed that red meats and sweets intake is an important factor which invariably aggravates the course of renal damage, induces hyperfiltration which, in turn can cause further renal damage as demonstrated in numerous animal studies [40]. We also found significant association between salt intake and progression of CKD which further support the theory.

Various thyroid functional disorders are frequently seen in CKD patients, resulting from alterations in thyroid hormone synthesis, metabolism, and regulation [41]. In our study $12.28 \%$ of CKD participants suffered from thyroid disease which suggests a significant correlation between the two. It is well known that age, education, hypertension, DM and cardiovascular disease are risk factors strongly correlated with CKD in addition to nephrotoxic medication. Acetaminophen and aspirin use in patients with newly diagnosed chronic renal failure was studied and found that regular use of either drug was associated with a 2.5 times greater CKD risk [42]. Similarly, it was observed that risks for CKD were higher for those who used second-generation antipsychotics (SGAs) [43] and statins [44]. While glucocorticoids has been extensively used in management of patients with CKD [45].

Overall, we found that the general public had poor levels of knowledge of CKD compared to the pharmacy graduates and nephrologists. These findings are in close agreement with reported results where public, students and nephrologists scores were 12, 19 and 23 (maximum score of 24), respectively [18]. Similarly, the mean (SD) knowledge score of Ethiopians participants was 11.12 ( \pm 4.21 ) with a minimum score of 0 and a maximum of 22 [97]. We found that $16.01 \%$ participants had good knowledge, while $19.22 \%$ and $64.73 \%$ had fair and poor knowledge, respectively. The values are low as observed by Oluyombo and coworkers where $27.1 \%$ had a good level of knowledge [20]. Overall, the results of this study showed poor understanding of CKD amongst the general public in Taif.

In our study we found that the occupation status of the respondents had significant effect on the knowledge of CKD. The respondents who were government employees had the highest mean knowledge score, followed by the respondents who were students, self-employed and non-employees. We didn't find significant relationship between age, gender, education and knowledge scores, and non-significant differences were observed with different education levels of education level.

Participants in this study had good knowledge about what CKD is (79.2\%) and the physiological role of the kidneys (87.9\%). More than half participants knew that person can lead a normal life with one kidney.

Participant knowledge about CKD risk factors and common symptoms was limited. Only few knew that diabetes (33.1\%) and high blood pressure (35.2\%) is responsible for CKD. In a hospital based cross-sectional study conducted in Ethiopia only 59 (28.4\%) of the participants had awareness about CKD and its risk factors. This study reported that $38.5 \%$ and $44.2 \%$ of participants knew hypertension and diabetes mellitus can cause CKD, respectively [46]. In a public survey of 748 participants conducted in Iran [47] only $12.7 \%$ and $14.4 \%$ knew that diabetes and hypertension were CKD risk factors; whereas a study of 516 community-dwelling Hong Kong adults reported that hypertension (43.8\%) and diabetes (44.0\%) as risk factors of kidney disease[98]. Only few (28.3\%) knew that CKD could be be inherited; whereas 
11.1\% in Nigeria agreed about the same factor [20]. Graduates and nephrologist were significantly associated with better knowledge score of risk factors for CKD.

When respondents were asked "Which of the following symptoms/signs that can manifest CKD", few have responded "bubbles in the urine" and "blood in the urine". Only 13.7\% identified that the "CKD can be asymptomatic until advanced stages" which was slightly higher (10.4\%) and slightly lower (17.8\%) than reported by Iranian and Chinese population, respectively [20,47]. More than half of the participants correctly identified edema or water retention as a symptom of advanced CKD. Regarding preventive measure for CKD, a high percentage agreed that healthy fluid intake in patient can prevent CKD, while few agreed with other healthy lifestyles such as keeping fit and healthy, controlling blood glucose and blood pressure.

Although the knowledge assessment survey was designed as a total scale score, there are some important items on the survey on which participants in this sample scored well. In particular, the sample had relatively greater knowledge (65\% or more correct) about function of the kidney, patients need to do screening, and patients need to be referred to nephrologists. Participants scored high on these knowledge items despite patients being unaware of the perceived causes, symptoms and risk factors of CKD. These results may reflect public awareness campaigns and national efforts to increase knowledge in this area. Hence, there is a need for a more targeted approach to increase the awareness and knowledge of CKD. Therefore, our study strongly suggests an organization and arrangement of various health campaigns to increase CKD knowledge among the general population.

\section{Conclusion}

Our study found various risk factors among CKD patients and a low level of patient awareness of chronic kidney disease in Taif, Saudi Arabia. The findings suggest that age $>50$ years, low educational attainment (illiterate or uncompleted primary school), family history of CKD, diabetes, hypertension, history of kidney stone, CVD and high BMI were significantly associated with CKD progression. Low physical activity, smoking, intake of low vegetables, fruits, fibers and high salt intake have been found to be a lifestyle and dietary factors that can contribute to the development of CKD. Similarly, the intake of certain nephrotoxic drugs may contribute to renal injury. Working toward increasing patient awareness and screening for CKD at regular intervals among high-risk groups is paramount to recognize chronic kidney disease at an early stage before it progresses to kidney failure helps to reduce the prevalence and mortality. We also found that the majority of the general public had poor knowledge of CKD compared to pharmacy graduates and nephrologists. These findings warrant the need for the development of awareness initiatives and programs designed to increase the level of knowledge of the general public of CKD.

\section{Compliance with ethical standards}

\section{Acknowledgments}

We have to express our appreciation to Dr. A.M.A. Hussein, Dr. Elwaleed Elmagzoub, Dr. Elsadig Al Yousif and Dr. Hussameldin Ali Hasan, Alhaada hospital for providing expertise that greatly assisted the research.

\section{Disclosure of conflict of interest}

The authors declare that there is no conflict of interest.

\section{Statement of informed consent}

Informed consent was obtained from all individual participants included in the study.

\section{References}

[1] Eknoyan G, Lameire N, Eckardt K, Kasiske B, Wheeler D, Levin A 2013. KDIGO 2012 clinical practice guideline for the evaluation and management of chronic kidney disease. Kidney Int Suppl. 3(1): 5-14.

[2] Damtie S, Biadgo B, Baynes HW, Ambachew S, Melak T, Asmelash D, Abebe M 2018. Chronic Kidney Disease and Associated Risk Factors Assessment among Diabetes Mellitus Patients at A Tertiary Hospital, Northwest Ethiopia. Ethiop J Health Sci. 28(6): 691-700.

[3] Schieppati A, Remuzzi G 2005. Chronic renal diseases as a public health problem: epidemiology, social, and economic implications. Kidney Int Suppl. 98: S7-S10. 
[4] Hajhosseiny R, Khavandi K, Goldsmith DJ 2013. Cardiovascular disease in chronic kidney disease: untying the Gordian knot. Int J Clin Pract. 67(1): 14-31.

[5] Wali RK 2010. Aspirin and the prevention of cardiovascular disease in chronic kidney disease: time to move forward? J Am Coll Cardiol. 56(12): 966-8.

[6] Alkerwi A, Sauvageot N, Bahi IE, Delagardelle C, Beissel J, Noppe J, Roderick PJ, Mindell JS, Stranges S 2017. Prevalence and related risk factors of chronic kidney disease among adults in Luxembourg: evidence from the observation of cardiovascular risk factors (ORISCAV-LUX) study. BMC Nephrol. 18: 358.

[7] Jha V, Garcia-Garcia G, Iseki K, Li Z, Naicker S, Plattner B, Saran R, Wang AY, Yang CW 2013. Chronic kidney disease: global dimension and perspectives. Lancet. 382(9888): 260-72.

[8] Go AS, Chertow GM, Fan D, McCulloch CE, Hsu CY 2004. Chronic kidney disease and the risks of death, cardiovascular events, and hospitalization. N Engl J Med. 351(13): 1296-305.

[9] Couser WG, Remuzzi G, Mendis S, Tonelli M 2011. The contribution of chronic kidney disease to the global burden of major noncommunicable diseases. Kidney Int. 80(12): 1258-70.

[10] Ho YW, Chau KF, Choy BY, Fung KS, Cheng YL, Kwan TH 2010. Hong Kong Renal Registry Report 2010. Hong Kong J. Nephrol . 12: 81-98.

[11] Hwang SJ, Tsai JC, Chen HC 2010. Epidemiology, impact and preventive care of chronic kidney disease in Taiwan. Nephrol. 15(2): 3-9.

[12] Barsoum RS 2006. Chronic kidney disease in the developing world. N Engl J Med. 354(10): 997-9.

[13] Duan J, Wang C, Liu D, Qiao Y, Shaokang Pan S, Jiang D, Zhao Z, Liang L, Tian F, Yu P, Zhang Y, Zhao H, Liu Z 2019. Prevalence and risk factors of chronic kidney disease and diabetic kidney disease in Chinese rural residents: a cross-sectional survey. Sci. Rep. 9: 10408.

[14] Zhang L, Zhang P, Wang F, Zuo L, Zhou Y, Shi Y, Li G, Jiao S, Liu Z, Liang W, Wang H 2008. Prevalence and factors associated with CKD: a population study from Beijing. Am J Kidney Dis. 51: 373-384.

[15] Goro KK, Wolide AD, Dibaba FK, Fufa FG, Garedow AW, Tufa BE, Bobasa EM 2019. Patient Awareness, Prevalence, and Risk Factors of Chronic Kidney Disease among Diabetes Mellitus and Hypertensive Patients at Jimma University Medical Center, Ethiopia. BioMed Res Int. 2383508

[16] Norton JM, Moxey-Mims MM, Eggers PW, Narva AS, Star RA, Kimmel PL, Griffin P. Rodgers GP 2016. Social determinants of racial disparities in CKD. Clin J Am Soc Nephrol. 27(9): 2576-2595, 2016.

[17] Bello AK, Peters Wight JJ, El Nahas M 2010. The kidney evaluation and awareness program in sheffield (KEAPS): a community-based screening for microalbuminuria in a british population. Nephron Clinical Practice. 116(2): c95-c103, 2010.

[18] Gheewala PA, Peterson GM, Zaidi STR, Matthew D. Jose MD, Castelino RL 2018. Public knowledge of chronic kidney disease evaluated using a validated questionnaire: a cross-sectional study. BMC Public Health. $18: 371$.

[19] Oluyombo R, Ayodele OE, Akinwusi PO, Okunola OO, Gbadegesin BA, Soje MO, Akinsola A 2016. Awareness, knowledge and perception of chronic kidney disease in a rural community of South-West Nigeria. Niger J Clin Pract. 19(2): 161-9.

[20] Chow KM, Szeto CC, Kwan B, Leung CB, Li PK 2014. Public lacks knowledge on chronic kidney disease: telephone survey. Hong Kong Med J. 20(2): 139-44.

[21] Ginawi IA, Ahmed HG, Al-hazimi AM. Assessment of Risk Factors for Chronic Kidney Disease in Saudi Arabia. International Journal of Science and ResearchVolume 3 Issue 7, July 2014, 446-450.

[22] Zeng X, Liu J, Tao S, Hong HG, Li Y, Fu P 2018. Associations between socioeconomic status and chronic kidney disease: a meta-analysis. J Epidemiol Community Health. 72(4): 270-279.

[23] Kazancioğlu R 2013. Risk factors for chronic kidney disease: an update. Kidney Int Suppl 2011. 3(4): 368-371.

[24] Drawz PE, Sedor JR, Hostetter TH. Family History and kidney Disease. AJKD, 2012, 59(1): 9-10.

[25] McClellan WM, Warnock DG, Judd S, Muntner P, Patzer RE, Bradbury BD, McClure LA, Newsome BB, Howard G 2012. Association of family history of ESRD, prevalent albuminuria, and reduced GFR with incident ESRD. Am J Kidney Dis. 59(1): 25-31. 
[26] Song EY, McClellan WM, McClellan A, Gadi R, Hadley AC, Krisher J, Clay M, Freedman BI 2009. Effect of community characteristics on familial clustering of end-stage renal disease. Am J Nephrol. 30(6): 499-504.

[27] Munkhaugen J, Lydersen S, Widerøe TE, Hallan S2009. Prehypertension, obesity, and risk of kidney disease: 20year follow-up of the HUNT I study in Norway. Am J Kidney Dis. 54(4): 638-46.

[28] Hsu CY, McCulloch CE, Iribarren C, Darbinian J, Go AS 2006. Body mass index and risk for end-stage renal disease. Ann Intern Med. 144(1): 21-8.

[29] Lu JL, Kalantar-Zadeh K, Ma JZ, Quarles LD, Kovesdy CP 2014. Association of body mass index with outcomes in patients with CKD. J Am Soc Nephrol. 25(9): 2088-96.

[30] Lai YJ, Hu HY, Lee YL, Ku PW, Yen YF, Chu D2017.Association between obesity and risk of chronic kidney disease: A nationwide Cohort study in Taiwan. NutrMetab Cardiovasc Dis. 27(11): 1008-1014.

[31] Lea JP, Nicholas SB 2002. Diabetes mellitus and hypertension: key risk factors for kidney disease. J Natl Med Assoc. 94(8 Suppl): 7S-15S.

[32] Süleymanlar G, Serdengeçti K, Altiparmak MR, Jager K, Seyahi N, Erek E; Turkish Registry of Nephrology, Dialysis, and Transplantation 2011. Trends in renal replacement therapy in Turkey, 1996-2008. Am J Kidney Dis. 57(3): 456-65.

[33] Parikh NI, Hwang SJ, Martin G. Larson MG. Cardiovascular Disease Risk Factors in Chronic Kidney Disease. Overall Burden and Rates of Treatment and Control. Arch Intern Med. 2006;166(17):1884-1891.

[34] Chen W, Chen W, Wang H, Dong X, L Qinghua, Mao H, Tan J, Lin J, Zhou F, Luo N, He H, Johnson RJ, Zhou SF, Yu S. Prevalence and risk factors associated with chronic kidney disease in an adult population from southern China. Nephrology Dialysis Transplantation, Volume 24, Issue 4, April 2009, Pages 1205-1212.

[35] Shang W, Li L, Ren Y, et al. History of kidney stones and risk of chronic kidney disease: a meta-analysis. PeerJ. 2017;5:e2907.

[36] Michishita R, Matsuda T, Kawakami S, Kiyonaga A, Tanaka H, Morito N, Higaki Y. The accumulation of healthy lifestyle behaviors prevents the incidence of chronic kidney disease (CKD) in middle-aged and older males. Environ Health Prev Med. 2016;21(3):129-137.

[37] Wakasugi M, Kazama JJ, Yamamoto S, Kawamura K, Narita I. A combination of healthy lifestyle factors is associated with a decreased incidence of chronic kidney disease: a population-based cohort study. Hypertens Res. 2013;36:328-333. Epidemiol. 2016 Jul 5;26(7):378-85. doi: 10.2188/jea.JE20150202. Epub 2016 Mar 5.

[38] Michishita R, Matsuda T, Kawakami S, Kiyonaga A, Tanaka H, Morito N, Higaki Y. The Association Between Unhealthy Lifestyle Behaviors and the Prevalence of Chronic Kidney Disease (CKD) in Middle-Aged and Older Men.

[39] Nettleton JA, Steffen LM, Palmas W, Burke GL, Jacobs DR Jr. Associations between microalbuminuria and animal foods, plant foods, and dietary patterns in the multiethnic study of atherosclerosis. Am J Clin Nutr. 2008;87:182536.

[40] Rysz J, Franczyk B, Ciałkowska-Rysz A, Gluba-Brzózka A. The Effect of Diet on the Survival of Patients with Chronic Kidney Disease. Nutrients. 2017;9(5):495.

[41] Rhee CM. The interaction between thyroid and kidney disease: an overview of the evidence. CurrOpin Endocrinol Diabetes Obes. 2016;23(5):407-415.

[42] Su T, Zhang L, Li X, Zuo L, Zhang P, Wang H. Regular use of nephrotoxic medications is an independent risk factor for chronic kidney disease-results from a Chinese population study. Nephrology Dialysis Transplantation, Volume 26, Issue 6, June 2011, Pages 1916-1923

[43] Wang HY, Huang CLC, Feng IJ, Hui-Chun Tsuang HC. Second-generation antipsychotic medications and risk of chronic kidney disease in schizophrenia: populationbased nested case-control study. BMJ Open 2018.

[44] Tonelli M, Lloyd AM, Bello AK, James MT, Klarenbach SW, McAlister FA, Manns BJ, Tsuyuki RT, Hemmelgarn B.Statin use and the risk of acute kidney injury in older adults. BMC Nephrology volume 20, Article number: 103 2019

[45] Ponticelli, C., \& Locatelli, F. 2018 Glucocorticoids in the treatment of glomerular diseases: pitfalls and pearls. Clinical Journal of the American Society of Nephrology, 13(5), 815-822. 
[46] KumelaGoro, K., Desalegn Wolide, A., Kerga Dibaba, F., GasheFufa, F., WakjiraGaredow, A., Edilu Tufa, B., \&MulisaBobasa, E. 2019. Patient Awareness, Prevalence, and Risk Factors of Chronic Kidney Disease among Diabetes Mellitus and Hypertensive Patients at Jimma University Medical Center, Ethiopia. BioMed Research International.

[47] Roomizadeh P, Taheri D, Abedini A, Mortazavi M, Larry M, Mehdikhani B, Mousavi SM, Hosseini FA, Parnia A, Nakhjavani M 2014. Limited knowledge of chronic kidney disease and its main risk factors among Iranian community: an appeal for promoting national public health education programs. Int J Health Policy Manag. 2(4): $161-166$. 